\title{
Localization and Orbital Selectivity in Iron-Based Superconductors with Cu Substitution
}

\author{
Yang Liu ${ }^{1}$, Da-Yong Liu ${ }^{2}$, Jiang-Long Wang ${ }^{3}$, Jian Sun ${ }^{1}$, Yun Song ${ }^{1}$. \\ ${ }^{1}$ Department of Physics, Beijing Normal University, Beijing 100875, China \\ ${ }^{2}$ Key Laboratory of Materials Physics, Institute of Solid State Physics, \\ Chinese Academy of Sciences, P. O. Box 1129, Hefei 230031, China and \\ ${ }^{3}$ College of Physics Science and Technology, Hebei University, Baoding 071002, China
}

(Dated: August 14, 2018)

\begin{abstract}
We study an inhomogeneous three-orbital Hubbard model for the Cu-substituted iron pnictides using an extended real-space Green's function method combined with density functional calculations. We find that the onsite interactions of the $\mathrm{Cu}$ ions are the principal determinant of whether an electron dopant or a hole dopant is caused by the $\mathrm{Cu}$ substitution. It is found that the $\mathrm{Cu}$ substitution could lead to a hole doping when its onsite interactions are smaller than a critical value, as opposed to an electron doping when the interactions of $\mathrm{Cu}$ ions are larger than the critical value, which may explain why the effects of $\mathrm{Cu}$ substitution on the carrier density are entirely different in $\mathrm{NaFe}_{1-x} \mathrm{Cu}_{x} \mathrm{As}$ and $\mathrm{Ba}\left(\mathrm{Fe}_{1-x} \mathrm{Cu}_{x}\right)_{2} \mathrm{As}_{2}$. We also find that the effect of a doping-induced disorder is considerable in the $\mathrm{Cu}$-substituted iron pnictides, and its cooperative effect with electron correlations contributes to the orbital-selective insulating phases in $\mathrm{NaFe}_{1-x} \mathrm{Cu}_{x} \mathrm{As}$ and $\mathrm{Ba}\left(\mathrm{Fe}_{1-x} \mathrm{Cu}_{x}\right)_{2} \mathrm{As}_{2}$.
\end{abstract}

\section{INTRODUCTION}

Iron-based superconductors ${ }^{1}-3$ have attracted great attention in recent years due to their considerable high superconducting transition temperatures and their rich phase diagrams, which are qualitatively similar to that of cuprate superconductors $\underline{4,5}$ In cuprate superconductors, substituting $\mathrm{Cu}$ with other transition metals is an effective way to gain a better insight into the origin of the high-temperature superconductivity. 6.7 Likewise, many experiments were conducted to reveal the effects of substituting $\mathrm{Fe}$ with other transition metals in some families of iron-based superconductors. -18 Among various transition-metal substitutions, $\mathrm{Cu}$ doping is highly disruptive to the electron structures of the FeAs sheets. There is lively debate on whether electron doping or hole doping occurs in Cu-substituted Fe-based superconductors $11-15$

On the one hand, the X-ray photoelectron spectra11,12 show that $\mathrm{Cu}$ has a closed $3 d^{10}$ shell in $\mathrm{Ba}\left(\mathrm{Fe}_{1-x} \mathrm{Cu}_{x}\right)_{2} \mathrm{As}_{2}$ and $\mathrm{SrFe}_{2-x} \mathrm{Cu}_{x} \mathrm{As}_{2}$ compounds, suggesting that $\mathrm{Cu}$ substitution behaves like hole doping. On the other hand, in the 122 phase, both $\mathrm{Cu}$ and $\mathrm{Ni}$ substitutions introduced a significant electron doping effect in $\mathrm{Ba}_{0.6} \mathrm{~K}_{0.4} \mathrm{Fe}_{2} \mathrm{As}_{2}$ and suppressed the superconducting transition temperature considerably 13 Nevertheless, the angle resolved photoemission spectroscopy (ARPES) experiment on $\mathrm{Ba}\left(\mathrm{Fe}_{1-x} \mathrm{Cu}_{x}\right)_{2} \mathrm{As}_{2}$ predicted that a part of the electrons in $\mathrm{Cu}$ substitution preferentially occupied the $\mathrm{Cu} 3 d$ states and did not behave like a mobile carrier. ${ }^{14.15}$ Moreover, the measurements on a series of $\mathrm{NaFe}_{1-x} \mathrm{Cu}_{x}$ As single crystals showed that $\mathrm{Cu}$ substitution serves as an effective electron dopant with strong impurity potential. ${ }^{16}$ Such an observation was also supported by the ARPES study, which exhibited the increasing of the Fermi level in $\mathrm{NaFe}_{1-x} \mathrm{Cu}_{x} \mathrm{As}$, indicating a fraction of electron doping introduced by the $\mathrm{Cu}$ dopant ${ }^{17} \mathrm{Up}$ to date, the dispute on the electron or hole doping in the 111 and 122 phases is far from resolved. Therefore, further investigations are needed to explain the reason why the $\mathrm{Cu}$ substitution plays different roles in $\mathrm{NaFe}_{1-x} \mathrm{Cu}_{x} \mathrm{As}$ and $\mathrm{Ba}\left(\mathrm{Fe}_{1-x} \mathrm{Cu}_{x}\right)_{2} \mathrm{As}_{2}$ compounds.

One also notices that the random distribution of $\mathrm{Cu}$ over Fe has been confirmed by the X-ray absorption nearedge structure in the Fe $K$ edge in $\mathrm{Fe}_{1-x} \mathrm{Cu}_{x} \mathrm{Se}_{1-\delta} .18$ The first-principles calculation also highlighted the necessity of including disorder effects on the iron-based superconductors with transition-metal substitution. ${ }^{19}$ Therefore, the quantum phase transition introduced by substituting $\mathrm{Fe}$ with $\mathrm{Cu}$ is complicated because $\mathrm{Cu}$ doping not only changes the carrier density but also leads to strong disorder effect. Additionally, the first-principles calculation demonstrated that the effective on-site Coulomb interactions in some families of Fe-based superconductors were considerably strong, 20 leading the parent compounds to be very close to the Mott insulating phase. ${ }^{21}$ The role of electronic correlation in the renormalizing of electronic bandwidths, ${ }^{22}$ magnetic moments ${ }^{23}$, orbitalselective Mott phase $, 24,25$ and orbital order 26 in ironpnictides is also recognized. More interestingly, heavy $\mathrm{Cu}$ doping drives the occurrence of a metal-insulator transition (MIT) ${ }^{16}$ which rarely happens in iron-pnictide compounds. This naturally raises a question as to whether the substitution of Fe with $\mathrm{Cu}$ drives the system to transit from a metal to an insulator, as was observed by Wang et al., 16 and what the nature of the MIT is.

To understand the mysterious electron or hole doping and the underlying physics of the MIT tuned by substituting $\mathrm{Fe}$ with $\mathrm{Cu}$ in an Fe-based superconductor, we proposed an inhomogeneous three-orbital Hubbard model after reconstructing the Fermi surfaces of NaFeAs and $\mathrm{NaCuAs}$ based on the first-principles calculations. Here we develop an extended real-space Green's function method, which is an adequate approach for the study of the cooperative effect of the multi-orbital electronic cor- 
relations and doping-induced disorder. We find that the increasing of the onsite interactions $u$ of the $\mathrm{Cu}$ ions can transfer electrons of $\mathrm{Cu}$ ions to $\mathrm{Fe}$ ions, and the critical point of the inversion from hole doping to electron doping is about $u_{c}=5.2 \mathrm{eV}$ when the interactions of Fe ions are $U=1.5 \mathrm{eV}$ and $J=U / 8$. It is found that the $\mathrm{Cu}$ substitutions lead to a hole doping when $u<5.2 \mathrm{eV}$, as opposed to an electron doping when $u \geq 5.2 \mathrm{eV}$, where the carrier occupancy of the $\mathrm{Cu}$ sites drops to zero. With the increasing of $\mathrm{Cu}$ substitutions, an enhancement of the Anderson localization in $d_{x z}$ and $d_{y z}$ orbitals is observed, whereas a Mott gap is found in the $d_{x y}$ orbital, indicating that the cooperative effect of multiorbital correlations and doping-induced disorder can lead to an orbitalselective insulating phase in the $\mathrm{Cu}$-substituted Fe-based superconductor.

This paper is organized as follows. In section II we first present the electronic structures of compounds $\mathrm{NaFe}_{1-x} \mathrm{Cu}_{x}$ As obtained by the first-principles calculations; we then propose an inhomogeneous three-orbital Hubbard model to study the cooperative effects of the multi-orbital correlations and doping-induced disorder in $\mathrm{Cu}$-substituted iron pnictides. The extended real-space Green's function approach employed in this paper is introduced in section [II]. In section IV, we study the effects of $\mathrm{Cu}$ substitution on the carrier density and discuss the orbital-selective insulating phase introduced by the cooperative effects of multi-orbital interactions and doping-induced disorder. The principal findings of this paper are summarized in section $\mathrm{V}$

\section{LDA BANDS AND MODEL HAMILTONIAN}

We first perform the local density functional calculation to understand the primary electronic structures of $\mathrm{Cu}$-doped compounds $\mathrm{NaFe}_{0.9} \mathrm{Cu}_{0.1} \mathrm{As}, \mathrm{NaFe}_{0.8} \mathrm{Cu}_{0.2} \mathrm{As}$, $\mathrm{NaFe}_{0.7} \mathrm{Cu}_{0.3} \mathrm{As}$, and $\mathrm{NaCuAs}$, as well as the parent compound NaFeAs. The band structures are calculated by the full potential local orbital (FPLO9) code 27 using the Perdew-Wang 92 version ${ }^{28}$ of the local-density approximation (LDA) for the exchange and correlation potentials. The orbitals of sodium $(2 s, 3 s, 4 s, 2 p, 3 p, 4 p$, and $3 d)$, iron $(3 s, 4 s, 5 s, 3 p, 4 p, 5 p, 3 d$, and $4 d)$, and arsenic $(3 s, 4 s, 5 s, 3 p, 4 p, 5 p, 3 d$, and $4 d)$ ions are treated as valence states, while the other lower-lying orbitals are considered as core states. Utilizing the lattice constants and atomic positions obtained by experiments, $\frac{16}{2}$ the selfconsistency process counts a total of 10416 irreducible $k$-points for the $k$-space integrations.

We plot the band structures of $\mathrm{NaFeAs}$ and $\mathrm{NaCuAs}$ in the upper and lower panels of Fig. 1. respectively. The heavy stripes represent the weights of $\mathrm{Fe}$ and $\mathrm{Cu} 3 d$ orbitals contributed to the bands. The weights are normalized, that is, the summation of the weights of all valence orbitals at a given point of the band structure should be unity. From Fig. 11, we find that the principal bands that determine the Fermi surfaces of NaFeAs comprise
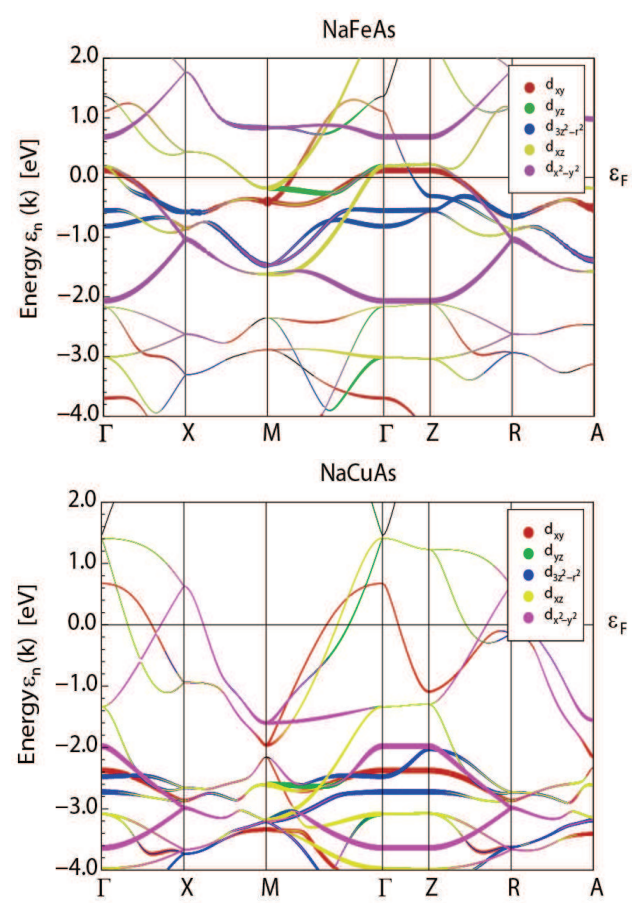

FIG. 1: (Color online) Band structures of NaFeAs (upper) and $\mathrm{NaCuAs}$ (lower) obtained by the GGA approach. Thick lines project out the $\mathrm{Cu} 3 d$ orbitals in the lower figure.

three strongly hybridized Fe $3 d$ orbitals $d_{x y}, d_{x z}$, and $d_{y z}$. Moreover, it is shown in Fig. 1 that the $\mathrm{Cu} 3 d$ orbitals primarily distribute from $-2 \mathrm{eV}$ to $-4 \mathrm{eV}$ below the Fermi level, suggesting that $3 d$ orbitals of doped $\mathrm{Cu}$ ions sink below Fermi level. In addition, we find that the weight of those bands around the Fermi level comes from As $4 p$ orbitals. The $\mathrm{Cu} 4 s$ and $\mathrm{Na} 3 s$ orbitals are higher in energy and irrelevant. We also plot the total density of states (DOS) of NaFeAs in Fig. 2, It is obvious that the DOS of Fe $3 d$ orbitals at the Fermi energy is dominant. While, the DOS of $\mathrm{Na} 3 s$ and As $4 p$ orbitals near the Fermi level are both very small, indicating that these orbitals could be ignored.

The coherent potential approximation $(\mathrm{CPA})^{29.30}$ is employed to account for the effect of substitutional disorder introduced by the $\mathrm{Cu}$ doping at $\mathrm{Fe}$ sites in $\mathrm{NaFe}_{1-x} \mathrm{Cu}_{x}$ As. As implemented in the FPLO5 code, $\stackrel{31}{=}$ the CPA approach treats approximately the disordered crystal as an effective medium 29.30 In the CPA calculation of the variation of electronic structure caused by $\mathrm{Cu}$ doping, the lattice parameters of $\mathrm{NaFe}_{1-x} \mathrm{Cu}_{x} \mathrm{As}(x=0.1$, 0.2 , and 0.3 ) are fixed at the experimental values of the parent compound $\mathrm{NaFeAs}$, and 1496 irreducible $k$-points are counted.

In Fig. 3, we show the DOS of $\mathrm{NaFe}_{1-x} \mathrm{Cu}_{x}$ As at $x=0.1$ and 0.3 near the Fermi level, respectively. With the increasing of $\mathrm{Cu}$-doping concentration, the energy distribution of $\mathrm{Cu} 3 d$ orbitals does not vary significantly, clearly indicating that the $\mathrm{Cu} 3 d$ orbitals always lie far below $\mathrm{E}_{F}$. We do not observe MIT over a wide region of $\mathrm{Cu}-$ 


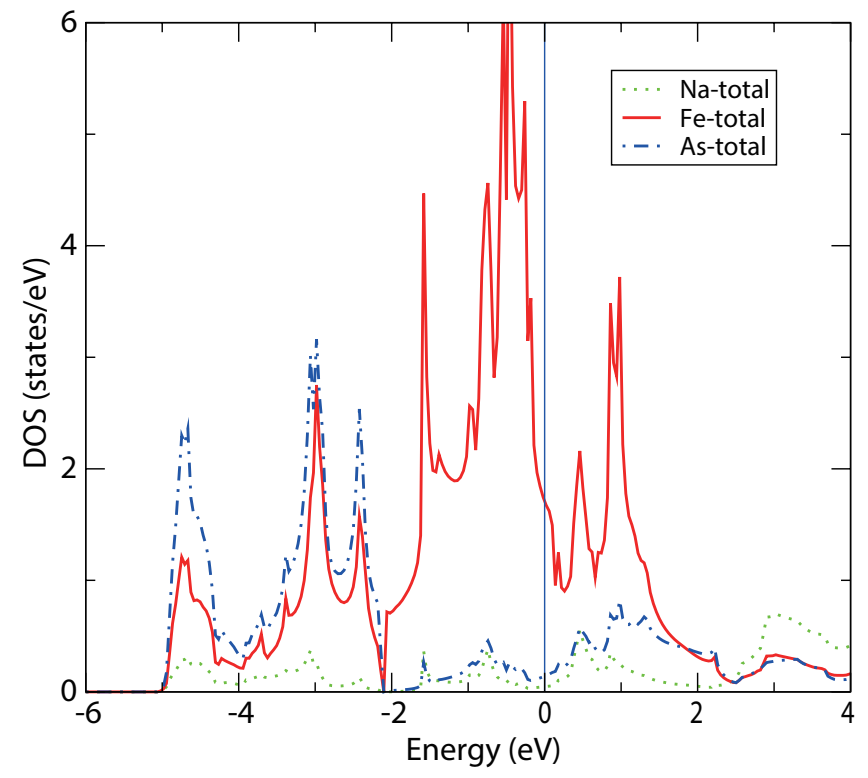

FIG. 2: (Color online) Density of states of NaFeAs obtained by the GGA approach. Three strongly hybridized Fe $3 d$ orbitals $d_{x y}, d_{x z}$, and $d_{y z}$ determine the Fermi surfaces of NaFeAs.

doping concentration, suggesting that the filling-factor controlled MIT could be ruled out, and thus the electronic correlation or disorder driven MIT is the most relevant candidate.

The band structure and orbital composition obtained by the first-principles calculation for undoped $\mathrm{NaFeAs}$ can be reproduced by a three-orbital tight-binding (TB) model. The TB model parameters are shown in Table [I which are very similar to the model parameters of Ref. [32]. Based on our LDA calculations of $\mathrm{NaFe}_{1-x} \mathrm{Cu}_{x} \mathrm{As}$, we introduce an inhomogeneous threeorbital Hubbard model for the $\mathrm{Cu}$-substituted Fe-based compounds, where a certain percentage of Fe sites replaced by $\mathrm{Cu}$ ions are chosen at random. Therefore, the whole Hamiltonian consists of three parts,

$$
H=H_{\mathrm{Fe}}+H_{\mathrm{Cu}}+H_{\mathrm{Hyb}},
$$

where $H_{\mathrm{Fe}}, H_{\mathrm{Cu}}$, and $H_{\mathrm{Hyb}}$ represent the Hamiltonians of Fe ions, $\mathrm{Cu}$-doping constitutions, and the hybridizations between $\mathrm{Fe}$ and $\mathrm{Cu}$ sites, respectively.

The partial Hamiltonian $H_{\mathrm{Fe}}$ for the electrons in $d_{x y}$, $d_{x z}$, and $d_{y z}$ orbitals of Fe ions can be expressed as

$$
\begin{aligned}
H_{\mathrm{Fe}}= & -\sum_{i \neq j(i, j \in F e)} \sum_{\alpha \beta \sigma} T_{i \alpha, j \beta} c_{i \alpha \sigma}^{\dagger} c_{j \beta \sigma} \\
& -\sum_{i \in F e, \alpha \sigma} \mu_{\alpha} n_{i \alpha \sigma}+U \sum_{i \in F e, \alpha} n_{i \alpha \uparrow} n_{i \alpha \downarrow} \\
& +\sum_{i \in F e} \sum_{\alpha<\beta, \sigma \sigma^{\prime}}\left(U^{\prime}-J \delta_{\sigma \sigma^{\prime}}\right) n_{i \alpha \sigma} n_{i \beta \sigma^{\prime}}
\end{aligned}
$$

where $c_{i \alpha \sigma}^{\dagger}\left(c_{i \alpha \sigma \sigma}\right)$ creates (annihilates) an electron with spin projection $\sigma$ for orbital $\alpha\left(d_{x z}, d_{y z}\right.$, or $\left.d_{x y}\right)$ of an iron

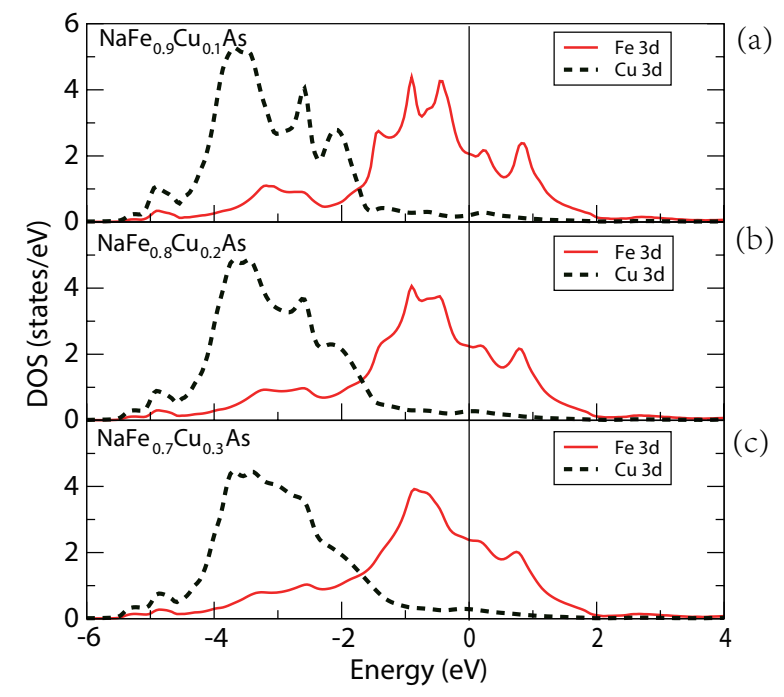

FIG. 3: (Color online) The density of states of compounds $\mathrm{NaFe}_{1-x} \mathrm{Cu}_{x}$ As at $x=0.1$ (a), $x=0.2$ (b), and $x=0.3$ (c) within the coherent potential approximation.

site $i$, and $n_{i \alpha \sigma}=c_{i \alpha \sigma}^{\dagger} c_{i \alpha \sigma}$ represents the corresponding electron number operator. $T_{i \alpha, j \beta}$ represent the hopping integrals of electrons between nearest-neighbor (NN) or next-nearest-neighbor (NNN) iron sites $i$ and $j$ within orbitals $\alpha$ and $\beta$ 른 $\Delta_{x y}=\mu_{x y}-\mu_{x z}$ is the energy difference between the $d_{x y}$ and degenerate $d_{x z} / d_{y z}$ orbitals. More details regarding the definition of kinetic energy in a Hamiltonian (2) can be found in appendix $\mathrm{A} U$ and $U^{\prime}$ are the on-site intra-orbital and inter-orbital Coulomb interactions on iron sites, respectively, and $J=J_{z}$ represents the Ising-type Hund's couplings. In our calculations, we ignore spin-flip and pair-hopping processes $\underline{34}-\underline{36}$

\begin{tabular}{|c|c|c|c|c|c|c|c|c|}
\hline$T_{1}$ & $T_{2}$ & $T_{3}$ & $T_{4}$ & $T_{5}$ & $T_{6}$ & $T_{7}$ & $T_{8}$ & $\Delta_{x y}$ \\
\hline & 0.15 & -0.12 & 0.06 & -0.08 & 0.1825 & 0.08375 & -0.03 & 0.75 \\
\hline
\end{tabular}

TABLE I: Parameters for the hopping integrals of the threeorbital Hubbard model ${ }^{32,33}$ The energy unit is electron volts. The quantities are defined in appendix A

As shown by the first-principles calculations, the hybridization is mainly between the $\mathrm{Fe} 3 d$ orbitals and the $\mathrm{Cu} 4 s$ orbitals, but it is very weak. The $\mathrm{Cu} 3 d$ orbitals distribute from $-2 \mathrm{eV}$ to $-4 \mathrm{eV}$ below the Fermi level, unlike the $\mathrm{Cu}$ ions in cuprate high- $T_{c}$ superconductors. When an Fe ion is substituted by a $\mathrm{Cu}$ ion in Fe-based superconductors, we need to consider all three $t_{2 g}$ orbitals of a $\mathrm{Cu}$ ion instead of the single $d_{x^{2}-y^{2}}$ orbital. Therefore, the Hamiltonian for the $\mathrm{Cu}$ substitutions is

$$
\begin{aligned}
H_{\mathrm{Cu}}= & -\sum_{i \neq j(i, j \in C u)} \sum_{\gamma \sigma} t_{i j} d_{i \gamma \sigma}^{\dagger} d_{j \gamma \sigma} \\
& -\sum_{i \in C u, \gamma \sigma} \nu_{\gamma} n_{i \gamma \sigma}^{d}+u \sum_{i \in C u, \gamma} n_{i \gamma \uparrow}^{d} n_{i \gamma \downarrow}^{d}
\end{aligned}
$$




$$
+\sum_{i \in C u} \sum_{\gamma<\lambda, \sigma \sigma^{\prime}}\left(u^{\prime}-j \delta_{\sigma \sigma^{\prime}}\right) n_{i \gamma \sigma}^{d} n_{i \lambda \sigma^{\prime}}^{d}
$$

where $d_{i^{\prime} \gamma \sigma}^{\dagger}\left(d_{i^{\prime} \gamma \sigma}\right)$ creates (annihilates) an electron at a copper site $i^{\prime} . \nu_{\gamma}$ are the chemical potentials of different orbitals $\gamma$. The intraorbital hopping integral between two copper sites is indicated as $t_{i^{\prime} j^{\prime}}$, and the intraorbital and interorbital interactions, as well as the Hund's rule couplings, are represented by $u, u^{\prime}$, and $j$ for copper sites, respectively.

As our calculations show that the intraorbital interactions $u$ play a principal role, only the effect of $u$ is taken into account. That is, we choose $u^{\prime}=u$ and $j=0$ in our discussion of $\mathrm{Cu}$ substitution effect in the iron pnictides. Because the $\mathrm{Cu} 3 d$ orbitals locate about $3 \mathrm{eV}$ below the Fermi level, the intraorbital hopping integrals $t_{i j}$ are predicted to be quite small. Owing to the restriction of the precision limitation of our calculations for the disordered cases, we approximate $t_{i j}$ in the range of 0 to $0.03 \mathrm{eV}$ for the $\mathrm{NN}$ hopping integrals between $\mathrm{Cu}$ ions. In our calculations, we choose $t=0.01 \mathrm{eV}$ for the NN hopping terms but $t=0$ for other long-distance hopping terms. We find that the value of $t$ only has an extremely-limited effect on the bandwidth of the $\mathrm{Cu}$ impurity band, and its effect on the DOS of Fe is negligible when the concentration of $\mathrm{Cu}$ substitution is less than 0.2 .

Moreover, the hybridization of $\mathrm{Fe}$ and $\mathrm{Cu}$ ions can be expressed as

$$
H_{\mathrm{Hyb}}=-\sum_{i \in F e, \alpha} \sum_{j \in C u, \gamma \sigma} t_{i j}^{\prime}\left(c_{i \alpha \sigma}^{\dagger} d_{j \gamma \sigma}+\text { H.c. }\right) \text {. }
$$

Because of the very weak effects of the long-distance terms, we only need to consider the NN hopping integrals $t^{\prime}$ between $\mathrm{Fe}$ and $\mathrm{Cu}$ ions, which are also assumed as $0.01 \mathrm{eV}$. Just the same as $t$, the effect of $t^{\prime}$ on the DOS of Fe ions is also negligible for the case with $x<0.2$. We study the inhomogeneous three-orbital Hubbard model Eq.(1) by developing a real-space Green's function method, which is described in the next section.

\section{REAL-SPACE GREEN'S FUNCTION APPROACH}

For a three-orbital Hubbard model, the real-space Green's function of a square lattice with $N=L^{2}$ sites is expressed as a $3 N \times 3 N$ matrix

$$
\mathscr{G}=\left(\begin{array}{ccccccc}
G_{11} & \cdots & G_{1, h-1} & F_{1 h}^{*} & G_{1, h+1} & \cdots & G_{1 M} \\
\vdots & \ddots & \vdots & \vdots & \vdots & \ddots & \vdots \\
G_{h-1,1} & \cdots & G_{h-1, h-1} & F_{h-1, h}^{*} & G_{h-1, h+1} & \cdots & G_{h-1, M} \\
F_{h 1} & \cdots & F_{h, h-1} & D_{h h} & F_{h, h+1} & \cdots & F_{h M} \\
G_{h+1,1} & \cdots & G_{h+1, h-1} & F_{h+1, h}^{*} & G_{h+1, h+1} & \cdots & G_{h+1, M} \\
\vdots & \ddots & \vdots & \vdots & \vdots & \ddots & \vdots \\
G_{M 1} & \cdots & G_{M, h-1} & F_{M h}^{*} & G_{M, h+1} & \cdots & G_{M M}
\end{array}\right)_{M=3 N}
$$

where $h$ represents the sites occupied by $\mathrm{Cu}$ ions. $G_{i j}$, $F_{i j}$, and $D_{i j}$ are $3 \times 3$ matrices with elements defined as

$$
\begin{aligned}
G_{i j}^{\alpha \beta} & =\left\langle\left\langle c_{i \alpha \sigma} \mid c_{j \beta \sigma}^{\dagger}\right\rangle\right\rangle \\
F_{i j}^{\alpha \beta} & =\left\langle\left\langle d_{i \alpha \sigma} \mid c_{j \beta \sigma}^{\dagger}\right\rangle\right\rangle \\
D_{i j}^{\alpha \beta} & =\left\langle\left\langle d_{i \alpha \sigma} \mid d_{j \beta \sigma}^{\dagger}\right\rangle\right\rangle .
\end{aligned}
$$

Each element of the Green's function matrices is obtained by the equation of motion, $\frac{37}{,}$ for example,

$$
\begin{aligned}
(\omega+ & \left.\mu_{\alpha}\right)\left\langle\left\langle c_{i \alpha \sigma} \mid c_{j \beta \sigma}^{\dagger}\right\rangle\right\rangle=\delta_{i j} \delta_{\alpha \beta}-\sum_{b \in C u, \gamma} t_{i b}^{\prime}\left\langle\left\langle d_{b \gamma \sigma} \mid c_{j \beta \sigma}^{\dagger}\right\rangle\right\rangle \\
& -\sum_{b \in F e, m} T_{i b \alpha m}\left\langle\left\langle c_{b m \sigma} \mid c_{j \beta \sigma}^{\dagger}\right\rangle\right\rangle \\
& +U\left\langle\left\langle n_{i \alpha \bar{\sigma}} c_{i \alpha \sigma} \mid c_{j \beta \sigma}^{\dagger}\right\rangle\right\rangle+U^{\prime} \sum_{l \neq \alpha}\left\langle\left\langle n_{i l \bar{\sigma}} c_{i \alpha \sigma} \mid c_{j \beta \sigma}^{\dagger}\right\rangle\right\rangle
\end{aligned}
$$

$$
+\left(U^{\prime}-J\right) \sum_{l \neq \alpha}\left\langle\left\langle n_{i l \sigma} c_{i \alpha \sigma} \mid c_{j \beta \sigma}^{\dagger}\right\rangle\right\rangle,
$$

from which come the second-order Green's functions, i.e. $\left\langle\left\langle n_{i l \bar{\sigma}} c_{i \alpha \sigma} \mid c_{j \beta \sigma}^{\dagger}\right\rangle\right\rangle$ and $\left\langle\left\langle n_{i l \sigma} c_{i \alpha \sigma} \mid c_{j \beta \sigma}^{\dagger}\right\rangle\right\rangle$ on the right side of Eq. (7). Similarly, the second-order Green's functions can be obtained by their equations of motion, where we will find the third-order Green's functions. Having an analogy with the Hubbard-I approximation,, 38 we introduce a proper decoupling process to make up a selfconsistent loop for the calculations of inhomogeneous Green's function $\hat{G}$. More details can be found in Appendix B.

We thus obtain a complete and solvable set of equations for all elements of the Green's function matrix $G$ in Eq.(5), 


$$
\begin{aligned}
M_{\alpha}\left\langle\left\langle c_{i \alpha \sigma} \mid c_{j \beta \sigma}^{\dagger}\right\rangle\right\rangle= & N_{\alpha} \delta_{i j} \delta_{\alpha \beta}-K_{\alpha} \delta_{i j} \delta_{l \beta}-\left(N_{\alpha}-K_{\alpha}\right)\left(\sum_{b \in F e, m} T_{i b \alpha m}\left\langle\left\langle c_{b m \sigma} \mid c_{j \beta \sigma}^{\dagger}\right\rangle\right\rangle+\sum_{b \in C u, \gamma} t_{i b}^{\prime}\left\langle\left\langle d_{b \gamma \sigma} \mid c_{j \beta \sigma}^{\dagger}\right\rangle\right\rangle\right) \\
& +\frac{U^{\prime}-J}{A_{\alpha}} \sum_{l \neq \alpha}\left(-\sum_{b \in F e, m} T_{i b l m}\left\langle c_{b m \sigma}^{\dagger} c_{i \alpha \sigma}\right\rangle+\sum_{b \in C u, \gamma} t_{i b}^{\prime}\left\langle d_{b \gamma \sigma}^{\dagger} c_{i \alpha \sigma}\right\rangle\right)\left\langle\left\langle c_{i l \sigma} \mid c_{j \beta \sigma}^{\dagger}\right\rangle\right\rangle, \\
M_{\alpha}\left\langle\left\langle c_{i \alpha \sigma} \mid d_{j \lambda \sigma}^{\dagger}\right\rangle\right\rangle= & -\left(N_{\alpha}-K_{\alpha}\right)\left(\sum_{b \in F e, m} T_{i b \alpha m}\left\langle\left\langle c_{b m \sigma} \mid d_{j \lambda \sigma}^{\dagger}\right\rangle\right\rangle+\sum_{b \in C u, \gamma} t_{i b}^{\prime}\left\langle\left\langle d_{b \gamma \sigma} \mid d_{j \lambda \sigma}^{\dagger}\right\rangle\right\rangle\right) \\
& +\frac{U^{\prime}-J}{A_{\alpha}} \sum_{l \neq \alpha}\left(-\sum_{b \in F e, m} T_{i b l m}\left\langle c_{b m \sigma}^{\dagger} c_{i \alpha \sigma}\right\rangle+\sum_{b \in C u, \gamma} t_{i b}^{\prime}\left\langle d_{b \gamma \sigma}^{\dagger} c_{i \alpha \sigma}\right\rangle\right)\left\langle\left\langle c_{i l \sigma} \mid d_{j \lambda \sigma}^{\dagger}\right\rangle\right\rangle, \\
\left(\omega+\nu_{\gamma}\right)\left\langle\left\langle d_{i \gamma \sigma} \mid c_{j \beta \sigma}^{\dagger}\right\rangle\right\rangle= & -\left(1+\frac{u\left\langle n_{i \bar{\sigma}}^{d}\right\rangle}{\omega+\nu_{\gamma}-u}\right)\left(\sum_{b \in C u} t_{i b}\left\langle\left\langle d_{b \gamma \sigma} \mid c_{j \beta \sigma}^{\dagger}\right\rangle\right\rangle+\sum_{a \in F e, \alpha} t_{a i}^{\prime}\left\langle\left\langle c_{a \alpha \sigma} \mid c_{j \beta \sigma}^{\dagger}\right\rangle\right\rangle\right), \\
\left(\omega+\nu_{\gamma}\right)\left\langle\left\langle d_{i \gamma \sigma} \mid d_{j \lambda \sigma}^{\dagger}\right\rangle\right\rangle= & \left(1+\frac{u\left\langle n_{i \bar{\sigma}}^{d}\right\rangle}{\omega+\nu_{\gamma}-u}\right)\left(\delta_{i j}-\sum_{b \in C u} t_{i b}\left\langle\left\langle d_{b \gamma \sigma} \mid d_{j \lambda \sigma}^{\dagger}\right\rangle\right\rangle-\sum_{a \in F e, \alpha} t_{a i}^{\prime}\left\langle\left\langle c_{a \alpha \sigma \sigma} \mid d_{j \lambda \sigma}^{\dagger}\right\rangle\right\rangle\right),
\end{aligned}
$$

with

$$
\begin{aligned}
M_{\alpha}= & \omega+\mu_{\alpha}+2 \sum_{b \in C u, \gamma} t_{i b}^{\prime}\left(B_{\alpha}\left\langle d_{b \gamma \bar{\sigma}}^{\dagger} c_{i \alpha \bar{\sigma}}\right\rangle\right. \\
& \left.+\frac{U^{\prime}-J}{A_{\alpha}} \sum_{l \neq \alpha, \gamma}\left\langle d_{b \gamma \sigma}^{\dagger} c_{i l \sigma}\right\rangle+\frac{U^{\prime}}{A_{\alpha}} \sum_{l \neq \alpha, \gamma}\left\langle d_{b \gamma \bar{\sigma}}^{\dagger} c_{i l \bar{\sigma}}\right\rangle\right), \\
N_{\alpha}= & 1+B_{\alpha}\left\langle n_{i \alpha \bar{\sigma}}\right\rangle+\frac{U^{\prime}-J}{A_{\alpha}} \sum_{l \neq \alpha}\left\langle n_{i l \sigma}\right\rangle+\frac{U^{\prime}}{A_{\alpha}} \sum_{l \neq \alpha}\left\langle n_{i l \bar{\sigma}}\right\rangle, \\
A_{\alpha}= & \omega+\mu_{\alpha}-U^{\prime} \sum_{s \neq \alpha, \sigma}\left\langle n_{i s \sigma}\right\rangle+J \sum_{s \neq \alpha}\left\langle n_{i s \sigma}\right\rangle, \\
B_{\alpha}= & \frac{U\left(1+\frac{U^{\prime}-J}{A_{\alpha}} \sum_{l \neq \alpha}\left\langle n_{i l \sigma}\right\rangle+\frac{U^{\prime}}{A_{\alpha}} \sum_{l \neq \alpha}\left\langle n_{i l \bar{\sigma}}\right\rangle\right)}{\omega+\mu_{\alpha}-U-U^{\prime} \sum_{l \neq \alpha, \sigma}\left\langle n_{i l \sigma}\right\rangle+J \sum_{l \neq \alpha}\left\langle n_{i l \sigma}\right\rangle}, \\
K_{\alpha}= & \frac{U^{\prime}-J}{A_{\alpha}} \sum_{l \neq \alpha}\left\langle c_{i l \sigma}^{\dagger} c_{i \alpha \sigma}\right\rangle .
\end{aligned}
$$

Therefore, the real-space Green's function matrix $\mathscr{G}$ can be obtained self-consistently by the matrix relationship

$$
\mathscr{M} \cdot \mathscr{G}=\mathscr{N}
$$

where $\mathscr{M}$ and $\mathscr{N}$ are also $3 N \times 3 N$ matrices that consist of the correlation functions, which can be obtained by the spectral theorem $\langle A B\rangle=-\frac{1}{\pi} \int_{-\infty}^{+\infty} f(\omega) \operatorname{Im}\langle\langle A \mid B\rangle\rangle$. The real-space Green's functions can be transformed into the momentum-space by using the Fourier transformation, $G_{\mathbf{k}}^{\alpha \beta}(\omega)=\frac{1}{N} \sum_{i j} G_{i j}^{\alpha \beta}(\omega) e^{-i \mathbf{k} \cdot\left(\mathbf{R}_{i}-\mathbf{R}_{j}\right)}$, where $\mathbf{k}$ denotes the wave-vectors in the unfolded Brillouin zone (BZ) $\underline{39}$ The one-particle spectral density is obtained by

$$
A(\mathbf{k}, \omega)=-\frac{1}{\pi} \operatorname{Im} G_{\mathbf{k}}(\omega),
$$

where the matrix of the momentum-space Green's function $G_{\mathbf{k}}(\omega)$ is diagonalized to include the strong hybridization of $d_{x y}, d_{x z}$ and $d_{y z}$ orbitals of Fe ions. In the next section, we study the effect of disorder introduced by substituting $\mathrm{Fe}$ with $\mathrm{Cu}$ in Fe-based superconductors.

To study the substitution effect of $\mathrm{Cu}$ ions in a finite square lattice, a portion of sites chosen randomly should be assigned to the $\mathrm{Cu}$ impurities. For example, if the concentration of the $\mathrm{Cu}$ substitution is $x=0.04$, we choose randomly 16 sites for $\mathrm{Cu}$ ions in a $N=20 \times 20$ lattice, and all the other sites are for Fe ions. For a certain disorder configuration, we calculate the real-space Green's function as described above to obtain physical properties, such as the local density of states (LDOS) at site $i$ for orbital $\alpha$,

$$
\rho_{\alpha}\left(\mathbf{r}_{i}, \omega\right)=-\frac{1}{\pi} \operatorname{Im} G_{i i}^{\alpha \alpha}(\omega)
$$

To find the averaged DOS of the whole system, we need to calculate the LDOS of different disorder configurations and then determine the sample-averaged values. The DOS of $\mathrm{Cu}$ and Fe ions can be expressed respectively as

$$
\begin{aligned}
& \rho_{\alpha}^{(F e)}=\frac{1}{N_{s}} \sum_{m} \frac{1}{N_{F e}} \sum_{i \in F e} \rho_{\alpha}^{(m)}\left(\mathbf{r}_{i}, \omega\right) \\
& \rho_{\alpha}^{(C u)}=\frac{1}{N_{s}} \sum_{m} \frac{1}{N_{C u}} \sum_{i \in C u} \rho_{\alpha}^{(m)}\left(\mathbf{r}_{i}, \omega\right),
\end{aligned}
$$

where $N_{F e}$ and $N_{C u}$ represent the total numbers of the Fe and $\mathrm{Cu}$ ions, respectively. $N_{s}$ is the number of disorder configurations, and we consider more than one hundred disorder samples in the following calculations. The effect of disorder could be studied accurately because our numerical method is just the same as the real-space exact diagonalization method ${ }^{41}$ when all the terms of interactions are omitted. 


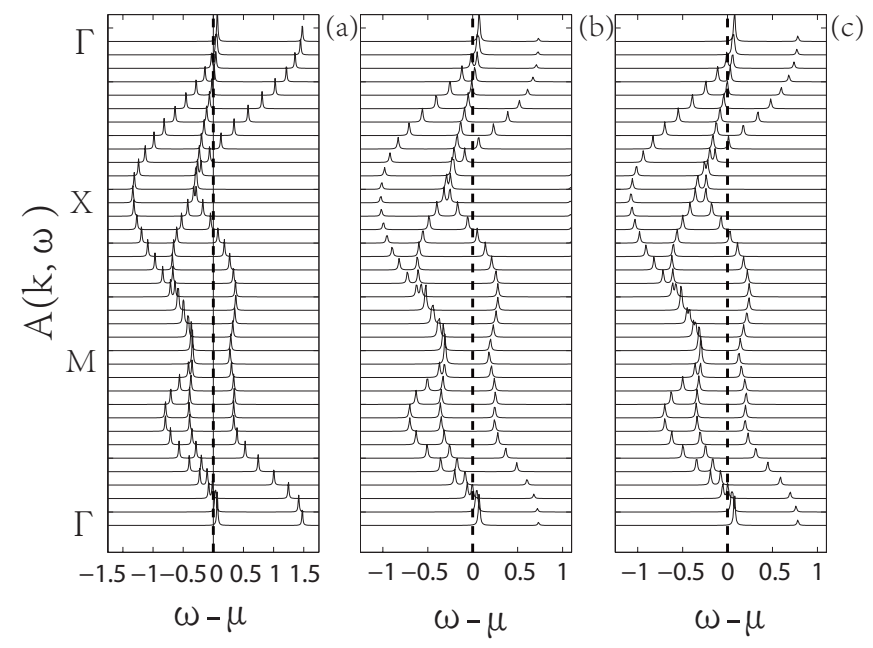

FIG. 4: Spectral density along the high-symmetry directions in the unfolded Brillouin zone with different intraorbital interactions: $U=0$ (a), $U=1 \mathrm{eV}(\mathrm{b})$, and $U=1.5 \mathrm{eV}$ (c) for undoped NaFeAs, where the Fermi energy is indicated by a dashed line. The Hund's rule coupling is $J=U / 8$, and $U^{\prime}=U-2 J$ is satisfied.

\section{COOPERATIVE EFFECT OF INTERACTIONS AND DISORDER}

The strong correlation and disorder, as described above, have been recognized as two possible fundamental origins that can drive the MIT. In the correlated fermion systems with the presence of disorder, the competition between Anderson and Mott-Hubbard MITs is still a theoretical challenge. $\stackrel{40}{ }$ Iron-based superconductors are typically multiorbital correlated materials. The ARPES measurement reported strong interactions between electrons in $\mathrm{NaFeAs} .42$ Furthermore, in $\mathrm{NaFe}_{1-x} \mathrm{Cu}_{x} \mathrm{As}$, a strong impurity potential is introduced by substituting Fe with $\mathrm{Cu}$ randomly ${ }^{16}$ In this section, to elucidate the $\mathrm{Cu}$ substitution effect in iron pnictides doped with copper, we use the newly developed real-space Green's function method described above to study the cooperative effect of the multi-orbital interactions and doping-induced disorder.

\section{A. Multi-orbital Interactions in undoped NaFeAs}

To estimate the multiorbital interaction parameters of Fe ions, such as the intraorbital interactions $U$, interorbital interactions $U^{\prime}$, and Hund's rule coupling $J$, we fit our numerical data of the band structures to some experimental and theoretical findings, as shown in Fig. 4. Because the undoped Fe-based superconductors are indicated to have a filling of approximately two-thirds based on the band structure calculations,,$\stackrel{43,44}{ }$ we determine the Fermi energy by using a constraint of four electrons per

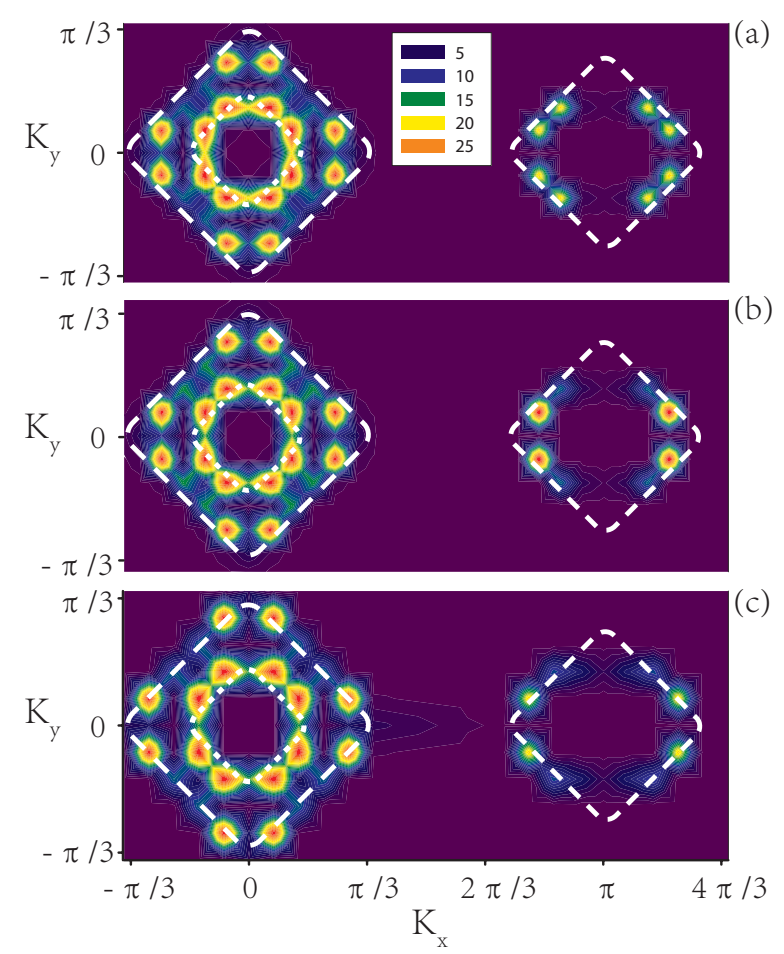

FIG. 5: (Color online) Fermi surfaces of the undoped threeorbital Hubbard model with $U=0$ (a), $U=1 \mathrm{eV}$ (b), and $U=1.5 \mathrm{eV}$ (c). In an area of the unfolded Brillouin zone, there are two hole pockets around the $\Gamma$ point $(0,0)$ and one electron pocket around the X point $(\pi, 0)$. The dashed lines show schematically the Fermi surfaces of the ARPES study on the parent compound of the 111 phase ${ }^{47,48}$ The model parameters are the same as those in Fig. 4

Fe,

$$
n_{\text {total }}=-\frac{1}{\pi} \sum_{i \alpha} \int_{-\infty}^{+\infty} f(\omega) \operatorname{Im} G_{i i}^{\alpha \alpha}(\omega) d \omega=4
$$

where $f(\omega)$ is the Fermi-Dirac distribution function.

Being consistent with the noninteracting spectral density of Ref. [32], all three $t_{2 g}$ bands across the Fermi surface with bandwidths of $D_{1}=1.95 \mathrm{eV}, D_{2}=0.9 \mathrm{eV}$, and $D_{3}=1.5 \mathrm{eV}$, respectively, are shown in Fig. 4(a) from high energy to low energy. A remarkable effect on the spectral function is found when the multi-orbital interactions are taken into account, especially when all three bands reduce their bandwidth with the increasing interactions. According to some previous theoretical studies of the iron pnictides, $, 32,33,45$ Hund's rule coupling is predicted to be $J=U / 8$ and the relation $U^{\prime}=U-2 J$ is also satisfied. As shown in Fig. 4 (c), the bandwidths of the three orbitals are $D_{1}=1 \mathrm{eV}, D_{2}=0.7 \mathrm{eV}$, and $D_{3}=1$ $\mathrm{eV}$, respectively, when $U$ is increased to $1.5 \mathrm{eV}$. Approximately, the on-site Coulomb repulsion renormalizes the bandwidth of the three bands across the Fermi surface 
by factors of $2,1.3$, and 1.5 , respectively, when $U=1.5$ $\mathrm{eV}$.

Compared with the ARPES measurement,, $40-50$ reasonable spectral functions and Fermi surfaces are reproduced qualitatively for undoped Fe-based superconductors NaFeAs and LiFeAs by using the numerical calculations of the undoped three-orbital Hubbard model. That is, when $U=1.5 \mathrm{eV}$, the bandwidths of the spectrum are about the half of the bandwidths obtained by the LDA, in good agreement with the ARPES experimental observation 50 Thus, the choice of the intraorbital interactions as $U=1.5 \mathrm{eV}$ is reasonable in the following numerical calculations of the $\mathrm{Cu}$ substituting effects in $\mathrm{NaFe}_{1-x} \mathrm{Cu}_{x} \mathrm{As}$.

Apart from the narrowing of the bandwidths, a significant shift of the Fermi level is also found accompanying the increasing interactions. The three-orbital tightbinding model exhibits two $d_{x z} / d_{y z}$-dominant hole pockets around the $\Gamma$ point and one $d_{x y}$-dominant electron pocket around the $\mathrm{X}$ point. The change of the interactions can only transfer electrons from $d_{x z} / d_{y z}$ orbitals to $d_{x y}$ orbital. With increasing $U$, a clear evidence of the increasing of the outer hole pocket is shown in Fig. 5. Accordingly, the sizes of the electron pocket and the inner hole pocket also expand with the increasing intraorbital interactions, but are not as significant as the size of the outer hole pocket. Our numerical results are qualitatively compatible with the LDA+DMFT study on the effects of interactions in LiFeAs. 51,52

For the convenience of comparison, we also display schematically the Fermi surfaces of ARPES study on the parent compound LiFeAs. ${ }^{47,48}$ As shown in Fig. 5(a) and Fig. 5(b), both the electron and the outer hole pockets obtained in the numerical simulations are smaller than the corresponding experimental results in either case, i.e. when $U=0$ or $U=1$. As shown in Fig. [5), we can find the best fitting of the Fermi surfaces obtained numerically with the experimental results, suggesting that the reasonable value of the onsite interactions of Fe ions should be $U=1.5 \mathrm{eV}$. Here we need to emphasize that, our theoretical model is applicable to both the 111 and 122 phases. As indicated by the ARPES experiments ${ }^{47}$, in $\mathrm{LiFeAs}$ and $\mathrm{BaFe}_{2} \mathrm{As}_{2}$, the sizes of the electron and hole pockets are comparable.

\section{B. Effect of $\mathrm{Cu}$ substitution on carrier density}

Some experiments concerning the effect of $\mathrm{Cu}$ substitution are mainly carried out in the 111-class and 122-class iron-based superconductors. In the 111 phase, such as $\mathrm{NaFe}_{1-x} \mathrm{Cu}_{x} \mathrm{As}^{17}$ and $\mathrm{LiFe}_{1-x} \mathrm{Cu}_{x} \mathrm{As},{ }^{53}$ it is found that $\mathrm{Cu}$ substitution enlarges the electron pocket and shrinks the hole pockets, implying that some extra electrons are brought in. Meanwhile, in the 122-phase, the experimental results have not reached agreement yet, but the prediction of hole doping, introduced by $\mathrm{Cu}$ substitution in 122 compounds, is better received. The $3 d$ states of a
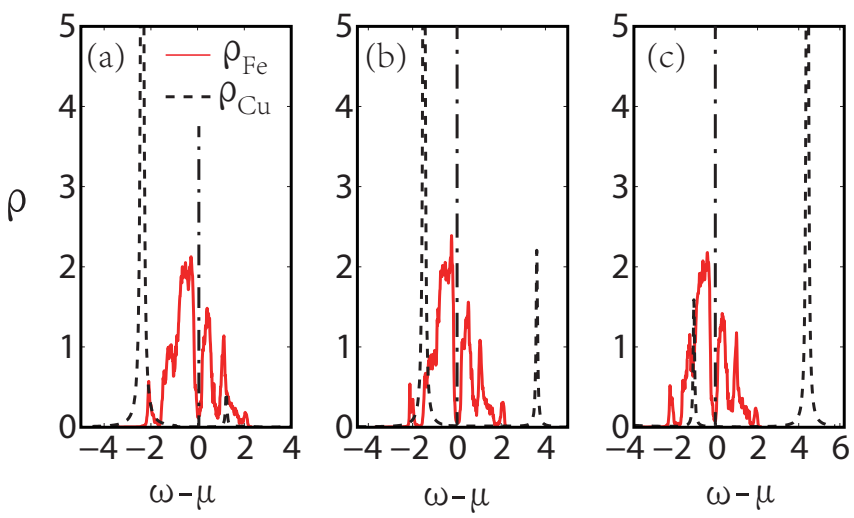

FIG. 6: (Color online) The average density of states of iron (solid line) and copper (dashed line) sites over 100 disorder configurations for different interactions of $\mathrm{Cu}$ ions: $u=3.6 \mathrm{eV}$ (a), $u=5.1 \mathrm{eV}$ (b), and $u=5.4 \mathrm{eV}$ (c). The intraorbital interactions of Fe ions are $U=1.5 \mathrm{eV}$, and the concentration of the $\mathrm{Cu}$ substitution is $x=0.04$. The lattice size is $N=20 \times 20$, and the total number of disorder samples is $N_{s}=100$.

$\mathrm{Cu}$ ion in $\mathrm{Ba}\left(\mathrm{Fe}_{1-x} \mathrm{Cu}_{x}\right)_{2} \mathrm{As}_{2}$ were found at the bottom of the valence band, forming a localized $3 d^{10}$ shell. ${ }^{12,54}$ As a result, the $\mathrm{Cu}$ substitution should result in a hole doping in the 122 phase $11,12,55,56$ Although the volume of the electron Fermi surface is smaller than the predicted value of the rigid-band model, effective electron doping introduced by the $\mathrm{Cu}$ substitutions was also predicted by other experiments. ${ }^{13,14}$

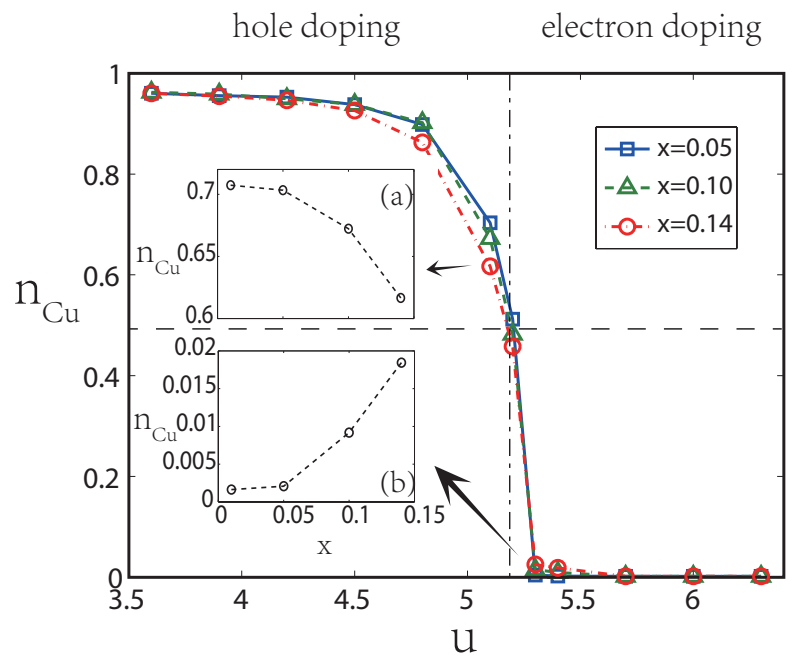

FIG. 7: (Color online) The average carrier occupancy on each copper site as a function of the interactions $u$. Inset: The substitution dependence of $n_{C u}$ for different interactions: $u=5.1$ $\mathrm{eV}$ (a), and $u=5.4 \mathrm{eV}$ (b) when $U=1.5 \mathrm{eV}$ and $J=U / 8$. The lattice sizes are $N=20 \times 20$, and one hundred disorder configurations $\left(N_{s}=100\right)$ are considered.

Next, we discuss the distinct effects of $\mathrm{Cu}$ substitutions on the carrier density in $\mathrm{NaFe}_{1-x} \mathrm{Fe}_{x} \mathrm{As}$ and $\mathrm{Ba}\left(\mathrm{Fe}_{1-x} \mathrm{Cu}_{x}\right)_{2} \mathrm{As}_{2}$. A first-principles calculation has 
indicated that the screened Coulomb interaction at $\mathrm{Fe}$ atoms of 122 families is less than that of Fe atoms of 111 families for approximately $0.35 \mathrm{eV} \stackrel{20}{2}$ As a result, for the two types of iron-based superconductors, the onsite interactions of the $\mathrm{Cu}$ impurities should be different accordingly. Because of the comparative localization of the $\mathrm{Cu}$ orbitals, the effective interactions of $\mathrm{Cu}$ ions should be considerably large. By contrast, the wave functions of $\mathrm{Fe}$ orbitals that cross the Fermi level are extended, introducing small effective interactions of Fe ions. For simplicity, in our calculation we assume that the interactions at $\mathrm{Cu}$ sites $(u)$ are in direct proportion to the interactions at $\mathrm{Fe}$ atoms $(U)$, with an approximate relation $u \simeq 4 U$. Considering that in Fe-based superconductors, the value of $U$ should be $1 \mathrm{eV}<U<2 \mathrm{eV}$, it is reasonable to predict the interactions of $\mathrm{Cu}$ ion as $u \approx 4-7 \mathrm{eV}$. The above estimated parameters have been proved to be appropriate by a combined valence band photoemission and Auger spectroscopy study of single crystalline $\mathrm{Ba}\left(\mathrm{Fe}_{1-x} \mathrm{Cu}_{x}\right)_{2} \mathrm{As}_{2}$, where the interactions in $\mathrm{Fe}$ and $\mathrm{Cu}$ ions are found to be $(1.4 \pm 0.6) \mathrm{eV}$ and $(7.5 \pm 0.4) \mathrm{eV}$, respectively $\underline{57}$ Assuming that $u \simeq 4 U$, the deviation is approximately $1.4 \mathrm{eV}$ for the $\mathrm{Cu}$ interactions of the 111 and 122 phases.

Effects of the onsite interactions of $\mathrm{Cu}$ ions on the DOS are shown in Fig. 6, where the concentration of $\mathrm{Cu}$ substitutions is fixed at $x=0.04$. For simplicity, the interactions of Fe ions are also fixed as $U=1.5 \mathrm{eV}$ and $J=U / 8$. When $u=3.6 \mathrm{eV}$, we find very narrow upper and lower Hubbard bands of $\mathrm{Cu}$ impurity that locate at $\omega=0.7$ $\mathrm{eV}$ and $\omega=-2.4 \mathrm{eV}$ respectively, as shown in Fig. 6(a). Furthermore, the DOS of lower Hubbard band is considerably larger than that of the upper Hubbard band, suggesting that most of the electrons in $\mathrm{Cu}$ ions locate far below the Fe bands when $u=3.6 \mathrm{eV}$. We also find that the DOS of the copper sites shifts from the lower Hubbard band to the upper band with increasing $u$ as shown in Fig. 6(b) and (c). In addition, the upper Hubbard band moves upwards, such as the upper Hubbard band raises to $\omega=4.3 \mathrm{eV}$ when $u=5.4 \mathrm{eV}$, which is far above the Fe bands. Our numerical results demonstrate that electrons in the $\mathrm{Cu}$ band can transfer to the $\mathrm{Fe}$ bands with the enhancement of the interactions of $\mathrm{Cu}$ ions.

As shown in Fig. 7 the carrier occupancy on $\mathrm{Cu}$ sites is obtained by integrating the corresponding DOS of $\mathrm{Cu}$ ions. We find that the average electron occupancy on $\mathrm{Cu}$ sites decreases with increasing $u$, especially the curve of $n_{\mathrm{Cu}}$ vs $u$, which drops sharply in the vicinity of $u=5.2$ $\mathrm{eV}$. From the perspective of the carrier density on the $\mathrm{Fe}$ sites, the $\mathrm{Cu}$ substitutions lead to a hole doping when $u<5.2 \mathrm{eV}$ because most electrons rest at the $\mathrm{Cu}$ orbitals. However, it is entirely different when $u \geq 5.2 \mathrm{eV}$, where the carrier occupancy of the $\mathrm{Cu}$ sites drops to zero, leading to electron doping.

To make it more clear, the inset figures in Fig. [7 show a reversal tendency of the dependence of $n_{\mathrm{Cu}}$ on $x$ for different $u$. It is shown that the electron occupancy of each $\mathrm{Cu}$ site decreases with increasing $x$ within the hole doping region with $u=5.1 \mathrm{eV}$. On the contrary, the occupancy increases with increasing $x$ in the electron doping region with $u=5.4 \mathrm{eV}$. The ARPES experiment on NaFeAs 17 indicates that the total number of electron carriers introduced by $\mathrm{Cu}$ atoms increases when the $\mathrm{Cu}$ substitution increases from 0.019 to 0.14 . However, the average number of electrons in $\mathrm{Cu}$ atom decreases significantly from 1.9 to 0.64 with increasing doping concentration. From the inset in Fig. 7(b), we find that the average electron occupancy on a single $\mathrm{Cu}$ site also increases with increasing $x$, implying that less electrons can be pushed from $\mathrm{Cu}$ sites to the iron sites. This tendency is consistent with the ARPES experiment.

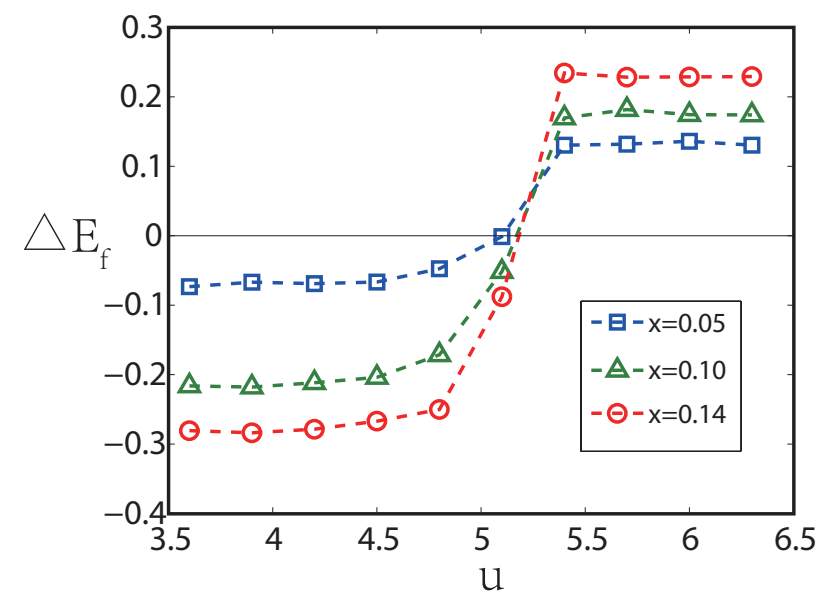

FIG. 8: (Color online) The variation of $\mathrm{E}_{f}, \Delta \mathrm{E}_{f}=E_{f}-E_{f 0}$, vs the interactions $u$ of $\mathrm{Cu}$ ions, where $E_{f 0}$ is the Fermi energy in undoped compound. The lattice parameters are the same as those in Fig. 7

In Fig. 8, we plot the dependence of the variation of the Fermi energy $\mathrm{E}_{f}$ on the interactions $u$. In the weak interaction range of $u$, the Fermi energy of a Cu-substituted compound is less than that of an undoped compound, being consistent with the hole doping feature. However, when $u>5.2 \mathrm{eV}, \Delta \mathrm{E}_{f}$ becomes positive, which means an electron doping appears. Our calculations demonstrate that the Coulomb interactions $u$ affect the average carrier density on iron sites and the Fermi energy of the whole system by pushing the electrons out of copper orbitals. For this reason, the different effects of $\mathrm{Cu}$ substitutions on Fermi energy and carrier density in 111 and 122 phases can be understood because the correlations of the 122 families are smaller than the correlations of the 111 families.

\section{Localization effects of $\mathrm{Cu}$ substitutions}

Aside from the change in carrier density, another remarkable effect of the $\mathrm{Cu}$ substitutions is the dopinginduced disorder. Adding the $\mathrm{Cu}$ substitutions, the bands are most strongly modified near the Fermi level. It is shown that both two hole pockets around the $\Gamma$ point 


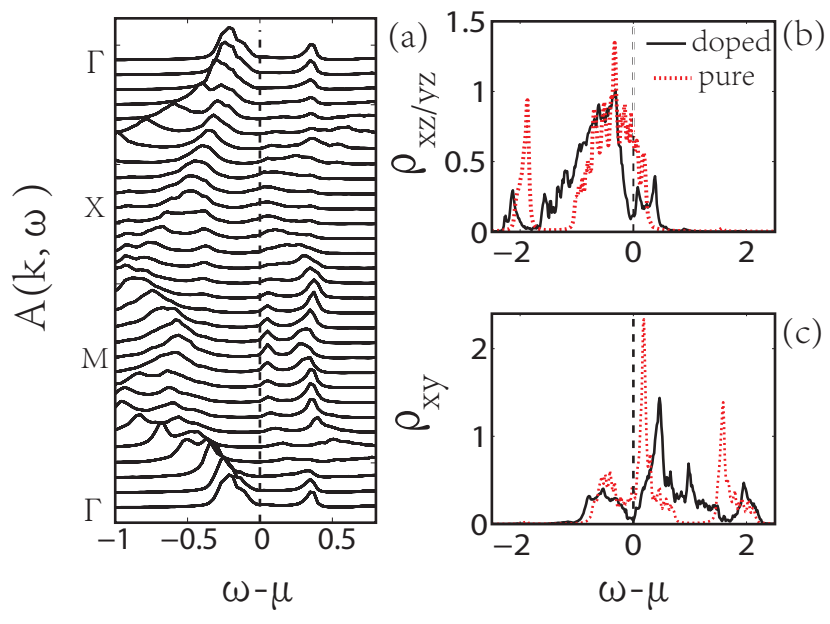

FIG. 9: (Color online) (a) Spectral density of Cu-doped NaFeAs with $\mathrm{Cu}$ impurity concentration $x=0.04$. (b) and (c) show the density of states of the degenerate $d_{x z} / d_{y z}$ and $d_{x y}$ orbitals for $x=0$ and $x=0.04$, respectively. The Fermi energy level is represented by the black dashed line. The lattice size is $N=20 \times 20$, and the number of disorder configurations is $N_{s}=100$. The other parameters are $U=1.5 \mathrm{eV}, J=U / 8$, and $u=6 \mathrm{eV}$.

and one electron pocket around the $X$ point are very sensitive to the $\mathrm{Cu}$ substitution. Apart from the shrinkage of the bandwidth, the most dramatic change in the band structures in $\mathrm{NaFe}_{1-x} \mathrm{Cu}_{x}$ As is that the spectral density near the Fermi surface is strongly suppressed, as shown in Fig. 9(a). As a result, the compound exhibits insulating or bad metallic behavior.

Efros and Shklovskii have demonstrated that the interactions between the localized electrons in a disordered system can create a Coulomb gap in the DOS near the Fermi level 58 As a result, the appearance of the zero-bias anomaly at the Fermi energy is seen as proof of the localization of electronic states in the correlated systems. 59 Just as expected, a soft gap can be found at the Fermi level in the DOS of both the degenerated $d_{x z} / d_{y z}$ orbitals and the $d_{x y}$ orbital when $x=0.04$, as shown in Fig. 9(b) and (c). The characteristic of the DOS of undoped compound is entirely different because there is no soft gap at Fermi level for all three orbitals, as shown by the red dashed lines in Fig. 9(b) and (c). Moreover, the EfrosShklovskii gap can also be found in Fig. 6. Therefore, we believe that the disorder effect in $\mathrm{NaFe}_{1-x} \mathrm{Cu}_{x} \mathrm{As}$ is considerably strong.

The iron-based superconductors are predicted to be close to the MIT border but are in the metallic side. 44 There are three possible origins for the MIT in the correlated system with disorder: (1) Mott-Hubbard MIT: the on-site Coulomb interactions split the energy band near the Fermi level into higher and lower Hubbard bands; (2) band occupation-driven MIT: changing the carrier density or bandwidth to make the conducting band fully filled; (3) Anderson MIT: electrons are localized in the real-space by Anderson localization effects introduced
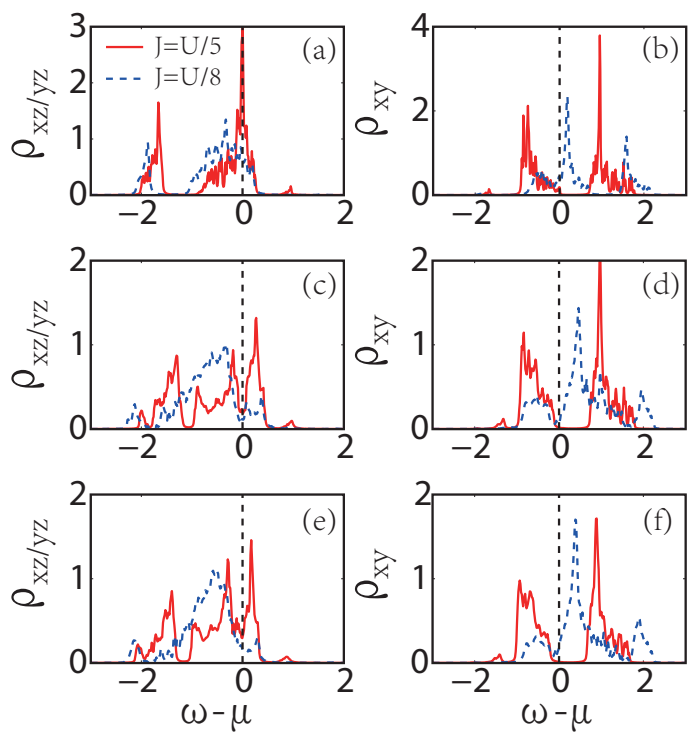

FIG. 10: (Color online) The evolution of the density of state of the $d_{x y}$ orbital (the left panel) and the $d_{x z} / d_{y z}$ orbitals (the right panel) for different $\mathrm{Cu}$ impurity concentrations: $x=0$ ((a) and (b)), $x=0.02((\mathrm{c})$ and (d)), and $x=0.08$ ((e) and (f)) when $J=U / 8$ (dashed line) and $J=U / 5$ (solid line), respectively. The other parameters are: $U=1.5 \mathrm{eV}$ and $u=6$ $\mathrm{eV}$. The lattice sizes are $N=20 \times 20$, and the number of disorder samples is $N_{s}=100$.

by disorder. Experiments found $\mathrm{Cu}$ substitution-driven MIT in $\mathrm{NaFe}_{1-x} \mathrm{Cu}_{x}$ As, but the mechanism of the MIT is still on debatable.

When $U=1.5 \mathrm{eV}$ and $J=\mathrm{U} / 8$, it is found that Anderson MIT happens with the increasing of the $\mathrm{Cu}$ substitutions, owing to the effect of disorder introduced by the $\mathrm{Cu}$ dopant in Fe-based superconductors. Besides, there is no Mott gap for both the $d_{x y}$ and $d_{x z} / d_{y z}$ orbitals at Fermi level when $J=U / 8$, as shown in Fig. 10. In general, the multi-orbital interactions, especially the Hund's rule couplings are likely to cause orbital-selective transitions ${ }^{24}$ It has also been predicted in some theoretical studies that a wide range of $J / U$ is possible for the iron-based superconductors. For example, the proper range of $J / U$ couplings was predicted as $0.1<J / U<0.33$, which was obtained by comparing the theoretical results with neutron scattering and ARPES experiments. $\frac{60}{}$ Therefore, we also study the effect of the Hund's rule coupling on the MIT in the $\mathrm{Cu}$-substituted iron pnictides. An orbitalselective insulating phase is found when we increase the Hund's rule coupling to $J=U / 5$ and the on-site interactions of $\mathrm{Cu}$ ions to $u=4 U=6 \mathrm{eV}$. By observing the evolution of the DOS of the three $3 d$ orbitals, we find that the properties of the insulating phase of the $d_{x z} / d_{y z}$ and $d_{x y}$ orbitals are different. As shown in the left panel of Fig. 10, a zero-bias anomaly dip can be found with increasing $\mathrm{Cu}$ substitution in the DOS of the $d_{x z} / d_{y z}$ orbital, but no hard gap can be obtained. Therefore, the lower-energy states at the Fermi level are Anderson localized as a result. Conversely, as shown in the right panel 
of Fig. 10, a hard Mott gap is opened in the Fermi level, indicating that the $d_{x y}$ orbital is in the Mott insulating state. In addition, when $J=U / 5$, the carrier type also changes from a hole doping to an electron doping with the increasing of the Coulomb interaction $u$ of $\mathrm{Cu}$ ions.

The effect of disorder on the conductance of Fe-based superconductors with $\mathrm{Cu}$ doping is studied by using the Kubo formula, $\frac{61,62}{6 h i c h}$ can be simplified, when $\omega=0$, as a $3 \times 3$ matrix $\sigma$ for the three orbital Hubbard model,

$$
\begin{aligned}
\sigma= & \frac{e^{2}}{\pi} \operatorname{Tr}\left[\operatorname{Im} G_{i}\left(j, j^{\prime}\right) \operatorname{Im} G_{i}\left(j^{\prime}-1, j-1\right)\right. \\
& +\operatorname{Im} G_{i}\left(j-1, j^{\prime}-1\right) \operatorname{Im} G_{i}\left(j^{\prime}, j\right) \\
& -\operatorname{Im} G_{i}\left(j, j^{\prime}-1\right) \operatorname{Im} G_{i}\left(j^{\prime}, j-1\right) \\
& \left.-\operatorname{Im} G_{i}\left(j-1, j^{\prime}\right) \operatorname{Im} G_{i}\left(j^{\prime}-1, j\right)\right]
\end{aligned}
$$

The trace is over the sites perpendicular to the current direction ( $k$ direction). $(i, j)$ denote the coordinates of lattice sites in the ribbon, where the periodic boundary conditions should be applied only to the $i$ direction, and $j$ and $j^{\prime}$ are chosen to be on opposite sides of the sample along the current direction. The conductance of the whole system can be obtained by adding the conductance of all three orbitals, which correspond to the three diagonal elements of the conductance matrix $\sigma$. As mentioned in Sec. III the averaged conductance is extracted by

$$
\bar{\sigma}=\frac{1}{N_{s}} \sum_{m} \sigma^{(m)},
$$

where $\sigma^{(m)}$ is the conductance obtained by Eq. (15) for the $\mathrm{m}$-th disorder configuration, and the resistance could be obtained directly by $\rho=1 / \bar{\sigma}$.

To compare with the experimental results, in Fig. 11, we show the temperature dependence of the resistance for different $\mathrm{Cu}$ concentrations. It is obvious that the compound behaves as an insulator when the density of the $\mathrm{Cu}$ substitution is over $x=0.1$. We find that the MIT in $\mathrm{NaFe}_{1-x} \mathrm{Cu}_{x}$ As is a transition from a metal to an orbital-selective insulating phase, which is caused by the cooperative effect of multi-orbital interactions and the doping-induced disorder. With the increasing of $\mathrm{Cu}$ substitutions, the effect of disorder significantly enhances, leading to strong Anderson localization in the $d_{x z}$ and $d_{y z}$ orbitals. Meanwhile, a Mott gap occurs in the $d_{x y}$ orbital. As a result, the resistance increases with increasing doping concentration both for the whole system and the individual orbitals, in good agreement with the experimental results. 63

\section{CONCLUSION}

We present the study of an extended real-space Green's function method combined with density function calculation on the cooperative effects of multiorbital correlations and disorder in $\mathrm{Cu}$-substituted iron-based superconductors. The inhomogeneous three-orbital Hub-
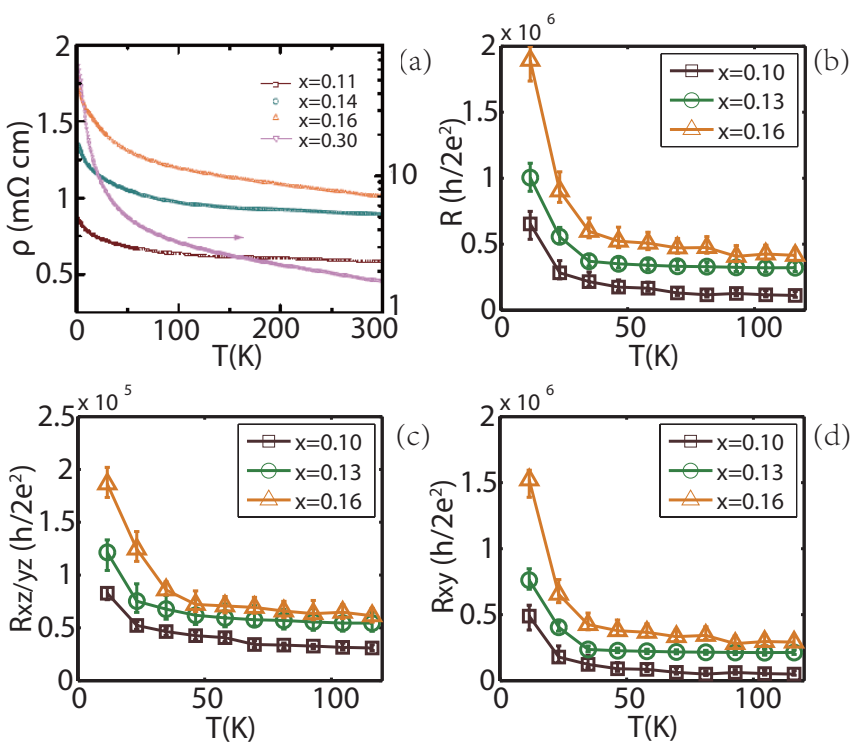

FIG. 11: (Color online) The temperature dependence of the resistance with increasing $\mathrm{Cu}$ substitution from $x=0.1$ to $x=0.16$. (a) Experimental data taken from Ref. [60]. (b) Numerical result of the resistance for the whole system. Additionally, the resistance of each orbital for $d_{x z} / d_{y z}$ (c) and $d_{x y}$ (d). Each data point is obtained by averaging for one hundred disorder configurations. The lattice sizes are $N=20 \times 20$, and one hundred disorder configurations $\left(N_{s}=100\right)$ are considered. The other model parameters are the same as in Fig. 10.

bard model has been employed to describe the substitution of $\mathrm{Cu}$ with $\mathrm{Fe}$ based on the electron structures of $\mathrm{NaFe}_{1-x} \mathrm{Cu}_{x} \mathrm{As}$ compounds. We find that the type of charge carriers introduced by the $\mathrm{Cu}$ substitution, namely, electrons or holes, is virtually determined by the effective interactions of the copper atoms. Due to the different environments surrounding the $\mathrm{Cu}$ substitution, the interactions of $\mathrm{Cu}$ ions in 111 phase are larger than that of 122 phase. As a result, a hole doping is introduced by the $\mathrm{Cu}$ substitution in $\mathrm{Ba}\left(\mathrm{Fe}_{1-x} \mathrm{Cu}_{x}\right){ }_{2} \mathrm{As}_{2}$, as opposed to an electron doping in $\mathrm{NaFe}_{1-x} \mathrm{Cu}_{x}$ As. Moreover, an orbital-selective insulating phase is observed where the $d_{x z}$ and $d_{y z}$ orbitals are Anderson localized, while the $d_{x y}$ orbital is in the Mott insulating phase. These results explain the experimental observations in $\mathrm{NaFe}_{1-x} \mathrm{Cu}_{x} \mathrm{As}$ and $\mathrm{Ba}\left(\mathrm{Fe}_{1-x} \mathrm{Cu}_{x}\right)_{2} \mathrm{As}_{2}$.

\section{Acknowledgments}

The computational resources utilized in this research were provided by Shanghai Supercomputer Center. The work was supported by the NSFC of China, under Grants No. 10974018, No. 11174036, No. 11474023, and No. 11274310, the National Basic Research Program of China (Grant No. 2011CBA00108), and the Fundamental Research Funds for the Central Universities. 
TABLE II: The NN and NNN hopping integrals of the electrons in the three orbitals $d_{x y}, d_{x z}$ and $d_{y z}$ of Fe ions. $(-1)^{|i|}$ indicates that the parameters change sign along the site locations.

\begin{tabular}{c|rrrrrrrr}
\hline \hline $\mathrm{T}_{i \alpha, j \beta}$ & \multicolumn{10}{c}{} \\
\hline$(\alpha, \beta) \backslash\left|\boldsymbol{R}_{i}-\boldsymbol{R}_{j}\right|$ & {$[1,0]$} & {$[0,1]$} & {$[-1,0]$} & {$[0,-1]$} & {$[1,1]$} & {$[-1,1]$} & {$[-1,-1]$} & {$[1,-1]$} \\
\hline$(x y, x y)$ & $T_{1}$ & $T_{1}$ & $T_{1}$ & $T_{1}$ & $T_{2}$ & $T_{2}$ & $T_{2}$ & $T_{2}$ \\
$(x y, x z)$ & $(-1)^{|i|} T_{3}$ & 0 & $(-1)^{|i+1|} T_{3}$ & 0 & $(-1)^{|i+1|} T_{4}$ & $(-1)^{|i|} T_{4}$ & $(-1)^{|i|} T_{4}$ & $(-1)^{|i+1|} T_{4}$ \\
$(x y, y z)$ & 0 & $(-1)^{|i|} T_{3}$ & 0 & $(-1)^{|i+1|} T_{3}$ & $(-1)^{|i+1|} T_{4}$ & $(-1)^{|i+1|} T_{4}$ & $(-1)^{|i|} T_{4}$ & $(-1)^{|i|} T_{4}$ \\
$(x z, x z)$ & $T_{6}$ & $T_{5}$ & $T_{6}$ & $T_{5}$ & $T_{7}$ & $T_{7}$ & $T_{7}$ & $T_{7}$ \\
$(y z, y z)$ & $T_{5}$ & $T_{6}$ & $T_{5}$ & $T_{6}$ & $T_{7}$ & $T_{7}$ & $T_{7}$ & $T_{7}$ \\
$(y z, x z)$ & 0 & 0 & 0 & 0 & $-T_{8}$ & $T_{8}$ & $-T_{8}$ & $T_{8}$ \\
\hline \hline
\end{tabular}

\section{Appendix A: Hopping integrals in the three-orbital tight-binding model}

As shown in Figs. 1 3, the local density function calculation predicted that the Fermi surface of $\mathrm{NaFe}_{1-x} \mathrm{Cu}_{x} \mathrm{As}$ composed only three Fe $3 d$ orbitals $\left(d_{x y}, d_{x z}\right.$ and $\left.d_{y z}\right)$, whereas $\mathrm{Cu} 3 d$ orbitals contributed from $-4 \mathrm{eV}$ to $-2 \mathrm{eV}$ under the Fermi level. (Eq. 2). Daghofer et al. have constructed a three-orbital Hamiltonian for the pnictides following the Slater-Koster procedure ${ }^{32,33}$ In keeping with their model, we mainly consider the $\mathrm{NN}$ and nextnearest-neighbor (NNN) hopping integrals of electrons in the three orbitals $d_{x y}, d_{x z}$, and $d_{y z}$ of iron ions. The definitions of the different types of hopping terms $T_{i \alpha, j \beta}$ in the tight-binding model are displayed in Table I.

The hopping integrals between the $d_{x y}$ and $d_{x z} / d_{y z}$ orbitals contain factors $(-1)^{i}$ that arise from the twoiron unit cell of the original FeAs planes ${ }^{33}$ As a result, those interorbital hopping terms change sign for $\mathrm{NN}$ iron sites. Moreover, the NN intraorbital hoppings between $d_{x y}$ and $d_{y z}$ along the $\hat{x}$ direction, and between $d_{x y}$ and $d_{x z}$ along the $\hat{y}$ direction, as well as the $\mathrm{NN}$ interorbital hopping between $d_{x z}$ and $d_{y z}$ orbitals, are all omitted.

Apart from the hopping terms, we need to add a term in the Hamiltonian for the energy splitting between the $d_{x y}$ and degenerate $d_{x z} / d_{y z}$ orbitals, $\Delta_{x y}$, which is crucial to providing the proper Fermi pockets when fitting with the local density approximation results.

\section{Appendix B: Decoupling approach}

All approximations that we used to decouple the thirdorder Green's functions to obtain Eq. (8) are listed as follows:

$$
\begin{aligned}
\left\langle\left\langle n_{i \alpha \sigma^{\prime}} c_{b m \sigma} \mid c_{j \beta \sigma}^{\dagger}\right\rangle\right\rangle & \approx\left\langle n_{i \alpha \sigma^{\prime}}\right\rangle\left\langle\left\langle c_{b m \sigma} \mid c_{j \beta \sigma}^{\dagger}\right\rangle\right\rangle, \\
\left\langle\left\langle n_{i \alpha \sigma^{\prime}} d_{b \gamma \sigma} \mid c_{j \beta \sigma}^{\dagger}\right\rangle\right\rangle & \approx\left\langle n_{i \alpha \sigma^{\prime}}\right\rangle\left\langle\left\langle d_{b \gamma \sigma} \mid c_{j \beta \sigma}^{\dagger}\right\rangle\right\rangle, \\
\left\langle\left\langle c_{b m \bar{\sigma}}^{\dagger} c_{i \alpha \bar{\sigma}} c_{i \alpha \sigma} \mid c_{j \beta \sigma}^{\dagger}\right\rangle\right\rangle & \approx\left\langle c_{b m \bar{\sigma}}^{\dagger} c_{i \alpha \bar{\sigma}}\right\rangle\left\langle\left\langle c_{i \alpha \sigma} \mid c_{j \beta \sigma}^{\dagger}\right\rangle\right\rangle, \\
\left\langle\left\langle c_{i \alpha \bar{\sigma}}^{\dagger} c_{b m \bar{\sigma}} c_{i \alpha \sigma} \mid c_{j \beta \sigma}^{\dagger}\right\rangle\right\rangle & \approx\left\langle c_{i \alpha \bar{\sigma}}^{\dagger} c_{b m \bar{\sigma}}\right\rangle\left\langle\left\langle c_{i \alpha \sigma} \mid c_{j \beta \sigma}^{\dagger}\right\rangle\right\rangle,
\end{aligned}
$$

$$
\begin{aligned}
\left\langle\left\langle c_{b m \bar{\sigma}}^{\dagger} c_{i l \bar{\sigma}} c_{i \alpha \sigma} \mid c_{j \beta \sigma}^{\dagger}\right\rangle\right\rangle \approx & \left\langle c_{b m \bar{\sigma}}^{\dagger} c_{i l \bar{\sigma}}\right\rangle\left\langle\left\langle c_{i \alpha \sigma} \mid c_{j \beta \sigma}^{\dagger}\right\rangle\right\rangle, \\
\left\langle\left\langle c_{i l \bar{\sigma}}^{\dagger} c_{b m \bar{\sigma}} c_{i \alpha \sigma} \mid c_{j \beta \sigma}^{\dagger}\right\rangle\right\rangle \approx & \left\langle c_{i l \bar{\sigma}}^{\dagger} c_{b m \bar{\sigma}}\right\rangle\left\langle\left\langle c_{i \alpha \sigma} \mid c_{j \beta \sigma}^{\dagger}\right\rangle\right\rangle, \\
\left\langle\left\langle c_{b m \sigma}^{\dagger} c_{i l \sigma} c_{i \alpha \sigma} \mid c_{j \beta \sigma}^{\dagger}\right\rangle\right\rangle \approx & \left\langle c_{b m \sigma}^{\dagger} c_{i l \sigma}\right\rangle\left\langle\left\langle c_{i \alpha \sigma} \mid c_{j \beta \sigma}^{\dagger}\right\rangle\right\rangle \\
& -\left\langle c_{b m \sigma}^{\dagger} c_{i \alpha \sigma}\right\rangle\left\langle\left\langle c_{i l \sigma} \mid c_{j \beta \sigma}^{\dagger}\right\rangle\right\rangle, \\
\left\langle\left\langle c_{i l \sigma}^{\dagger} c_{b m \sigma} c_{i \alpha \sigma} \mid c_{j \beta \sigma}^{\dagger}\right\rangle\right\rangle \approx & \left\langle c_{i l \sigma}^{\dagger} c_{b m \sigma}\right\rangle\left\langle\left\langle c_{i \alpha \sigma} \mid c_{j \beta \sigma}^{\dagger}\right\rangle\right\rangle \\
& -\left\langle c_{i l \sigma}^{\dagger} c_{i \alpha \sigma}\right\rangle\left\langle\left\langle c_{b m \sigma} \mid c_{j \beta \sigma}^{\dagger}\right\rangle\right\rangle, \\
\left\langle\left\langle d_{b \gamma \bar{\sigma}}^{\dagger} c_{i \alpha \bar{\sigma}} c_{i \alpha \sigma} \mid c_{j \beta \sigma}^{\dagger}\right\rangle\right\rangle \approx & \left\langle d_{b \gamma \bar{\sigma}}^{\dagger} c_{i \alpha \bar{\sigma}}\right\rangle\left\langle\left\langle c_{i \alpha \sigma} \mid c_{j \beta \sigma}^{\dagger}\right\rangle\right\rangle, \\
\left\langle\left\langle c_{i \alpha \bar{\sigma}}^{\dagger} d_{b \gamma \bar{\sigma}} c_{i \alpha \sigma} \mid c_{j \beta \sigma}^{\dagger}\right\rangle\right\rangle \approx & \left\langle c_{i \alpha \bar{\sigma}}^{\dagger} d_{b \gamma \bar{\sigma}}\right\rangle\left\langle\left\langle c_{i \alpha \sigma} \mid c_{j \beta \sigma}^{\dagger}\right\rangle\right\rangle, \\
\left\langle\left\langle d_{b \gamma \bar{\sigma}}^{\dagger} c_{i l \bar{\sigma}} c_{i \alpha \sigma} \mid c_{j \beta \sigma}^{\dagger}\right\rangle\right\rangle \approx & \left\langle d_{b \gamma \bar{\sigma}}^{\dagger} c_{i l \bar{\sigma}}\right\rangle\left\langle\left\langle c_{i \alpha \sigma} \mid c_{j \beta \sigma}^{\dagger}\right\rangle\right\rangle, \\
\left\langle\left\langle c_{i l \bar{\sigma}}^{\dagger} d_{b \gamma \bar{\sigma}} c_{i \alpha \sigma} \mid c_{j \beta \sigma}^{\dagger}\right\rangle\right\rangle \approx & \left\langle c_{i l \bar{\sigma}}^{\dagger} d_{b \gamma \bar{\sigma}}\right\rangle\left\langle\left\langle c_{i \alpha \sigma} \mid c_{j \beta \sigma}^{\dagger}\right\rangle\right\rangle, \\
\left\langle\left\langle d_{b \gamma \sigma}^{\dagger} c_{i l \sigma} c_{i \alpha \sigma} \mid c_{j \beta \sigma}^{\dagger}\right\rangle\right\rangle \approx & \left\langle d_{b \gamma \sigma}^{\dagger} c_{i l \sigma}\right\rangle\left\langle\left\langle c_{i \alpha \sigma} \mid c_{j \beta \sigma}^{\dagger}\right\rangle\right\rangle \\
& -\left\langle d_{b \gamma \sigma}^{\dagger} c_{i \alpha \sigma}\right\rangle\left\langle\left\langle c_{i l \sigma} \mid c_{j \beta \sigma}^{\dagger}\right\rangle\right\rangle, \\
\left\langle\left\langle n_{i s \sigma^{\prime}} n_{i l \sigma^{\prime \prime}} c_{i \alpha \sigma} \mid c_{j \beta \sigma}^{\dagger}\right\rangle\right\rangle \approx & \left\langle n_{i s \sigma^{\prime}}\right\rangle\left\langle\left\langle n_{i l \sigma^{\prime \prime}} c_{i \alpha \sigma} \mid c_{j \beta \sigma}^{\dagger}\right\rangle\right\rangle \\
& +\left\langle n_{i l \sigma^{\prime \prime}}\right\rangle\left\langle\left\langle n_{i s \sigma^{\prime}} c_{i \alpha \sigma} \mid c_{j \beta \sigma}^{\dagger}\right\rangle\right\rangle, \\
\left\langle\left\langle n_{i l \sigma}^{\dagger} d_{b \gamma \sigma} c_{i \alpha \sigma} \mid c_{j \beta \sigma}^{\dagger}\right\rangle\right\rangle \approx & \left\langle c_{i l \sigma}^{\dagger} d_{b \gamma \sigma}\right\rangle\left\langle\left\langle c_{i \alpha \sigma} \mid c_{j \beta \sigma}^{\dagger}\right\rangle\right\rangle \\
& -\left\langle c_{i l \sigma}^{\dagger} c_{i \alpha \sigma}\right\rangle\left\langle\left\langle d_{b \gamma \sigma} \mid c_{j \beta \sigma}^{\dagger}\right\rangle\right\rangle, \\
& \left\langle n_{i l \sigma^{\prime}}\right\rangle\left\langle\left\langle n_{i \alpha \bar{\sigma}} c_{i \alpha \sigma} \mid c_{j \beta \sigma}^{\dagger}\right\rangle\right\rangle, \\
\left.n_{i \alpha \bar{\sigma}} c_{i \alpha \sigma}\left|c_{j \beta \sigma}\right\rangle\right\rangle \approx &
\end{aligned}
$$

where $\sigma^{\prime}$ and $\sigma^{\prime \prime}$ represent $\sigma$ or $\bar{\sigma}$.

In the above decoupling scheme, we follow the standard decoupling method ${ }^{64}$ and introduce three basic rules for the multi-orbital model as: (1) Some specific secondorder Green's functions, such as $\left\langle\left\langle n_{i \alpha \bar{\sigma}} c_{i \alpha \sigma} \mid c_{j \beta \sigma}^{\dagger}\right\rangle\right\rangle$, $\left\langle\left\langle n_{i l \sigma} c_{i \alpha \sigma} \mid c_{j \beta \sigma}^{\dagger}\right\rangle\right\rangle$, and $\left\langle\left\langle n_{i l \bar{\sigma}} c_{i \alpha \sigma} \mid c_{j \beta \sigma}^{\dagger}\right\rangle\right\rangle$, are regarded as basic Green's functions that receive the same treatment as the first-order Green's function. That is, we also need to find the equations of motions of those Green's functions. (2) In a third-order Green's function with $n$ operators, such as $\left\langle\left\langle n_{i l \sigma^{\prime}} n_{i \alpha \bar{\sigma}} c_{i \alpha \sigma} \mid c_{j \beta \sigma}^{\dagger}\right\rangle\right\rangle$, we introduce the average value of $\left\langle n_{i l \sigma^{\prime}}\right\rangle$ to get the lower-order Green's function as $\left\langle n_{i l \sigma^{\prime}}\right\rangle\left\langle\left\langle n_{i \alpha \bar{\sigma}} c_{i \alpha \sigma} \mid c_{j \beta \sigma}^{\dagger}\right\rangle\right\rangle$ because its orbital index $l$ is different from $\alpha$ of the other operators. (3) We 
do not consider the effects of the correlation functions that contain spin flip terms. As a result, the equations of motion of some basic second-order Green's functions can also be obtained as

$$
\begin{aligned}
& \left(\omega+\mu_{\alpha}-U_{1}-U^{\prime} \sum_{l \neq \alpha}\left\langle n_{i l}\right\rangle+J_{z} \sum_{l \neq \alpha}\left\langle n_{i l \sigma}\right\rangle\right)\left\langle\left\langle n_{i \alpha \bar{\sigma}} c_{i \alpha \sigma} \mid c_{j \beta \sigma}^{\dagger}\right\rangle\right\rangle=\left\langle n_{i \alpha \bar{\sigma}}\right\rangle \delta_{i j} \delta_{\alpha \beta}-\sum_{b \in F e, m} T_{i b \alpha m}\left\langle n_{i \alpha \bar{\sigma}}\right\rangle\left\langle\left\langle c_{b m \sigma} \mid c_{j \beta \sigma}^{\dagger}\right\rangle\right\rangle \\
& -\sum_{b \in C u} t_{i b}^{\prime}\left(2\left\langle d_{b \bar{\sigma}}^{\dagger} c_{i \alpha \bar{\sigma}}\right\rangle\left\langle\left\langle c_{i \alpha \sigma} \mid c_{j \beta \sigma}^{\dagger}\right\rangle\right\rangle+\left\langle n_{i \alpha \bar{\sigma}}\right\rangle\left\langle\left\langle d_{b \sigma} \mid c_{j \beta \sigma}^{\dagger}\right\rangle\right\rangle\right), \\
& \left(\omega+\mu_{\alpha}-U^{\prime} \sum_{s \neq \alpha}\left\langle n_{i s}\right\rangle+J_{z} \sum_{s \neq \alpha}\left\langle n_{i s \sigma}\right\rangle\right)\left\langle\left\langle n_{i l \sigma} c_{i \alpha \sigma} \mid c_{j \beta \sigma}^{\dagger}\right\rangle\right\rangle=\left\langle n_{i l \sigma}\right\rangle \delta_{i j} \delta_{\alpha \beta}-\left\langle c_{i l \sigma}^{\dagger} c_{i \alpha \sigma}\right\rangle \delta_{i j} \delta_{l \beta} \\
& -\sum_{b \in F e, m} T_{i b l m}\left(\left\langle c_{b m \sigma}^{\dagger} c_{i \alpha \sigma}\right\rangle\left\langle\left\langle c_{i l \sigma} \mid c_{j \beta \sigma}^{\dagger}\right\rangle\right\rangle-\left\langle c_{i l \sigma}^{\dagger} c_{i \alpha \sigma}\right\rangle\left\langle\left\langle c_{b m \sigma} \mid c_{j \beta \sigma}^{\dagger}\right\rangle\right\rangle\right)-\sum_{b \in F e, m} T_{i b \alpha m}\left\langle n_{i l \sigma}\right\rangle\left\langle\left\langle c_{b m \sigma} \mid c_{j \beta \sigma}^{\dagger}\right\rangle\right\rangle \\
& \left.-\sum_{b \in C u} t_{i b}^{\prime}\left[\left(\left\langle n_{i l \sigma}\right\rangle-\left\langle c_{i l \sigma}^{\dagger} c_{i \alpha \sigma}\right\rangle\right)\left\langle\left\langle d_{b \sigma} \mid c_{j \beta \sigma}^{\dagger}\right\rangle\right\rangle\right)+2\left\langle d_{b \sigma}^{\dagger} c_{i l \sigma}\right\rangle\left\langle\left\langle c_{i \alpha \sigma} \mid c_{j \beta \sigma}^{\dagger}\right\rangle\right\rangle-\left\langle d_{b \sigma}^{\dagger} c_{i \alpha \sigma}\right\rangle\left\langle\left\langle c_{i \alpha \sigma} \mid c_{j \beta \sigma}^{\dagger}\right\rangle\right\rangle\right] \\
& +U_{1}\left\langle n_{i l \sigma}\right\rangle\left\langle\left\langle n_{i \alpha \bar{\sigma}} c_{i \alpha \sigma} \mid c_{j \beta \sigma}^{\dagger}\right\rangle\right\rangle \\
& \left(\omega+\mu_{\alpha}-U^{\prime} \sum_{s \neq \alpha}\left\langle n_{i s}\right\rangle+J_{z} \sum_{s \neq \alpha}\left\langle n_{i s \sigma}\right\rangle\right)\left\langle\left\langle n_{i l \bar{\sigma}} c_{i \alpha \sigma} \mid c_{j \beta \sigma}^{\dagger}\right\rangle\right\rangle=\left\langle n_{i l \bar{\sigma}}\right\rangle \delta_{i j} \delta_{\alpha \beta}-\sum_{b \in F e, m} T_{i b \alpha m}\left\langle n_{i l \bar{\sigma}}\right\rangle\left\langle\left\langle c_{b m \sigma} \mid c_{j \beta \sigma}^{\dagger}\right\rangle\right\rangle \\
& +U_{1}\left\langle n_{i l \bar{\sigma}}\right\rangle\left\langle\left\langle n_{i \alpha \bar{\sigma}} c_{i \alpha \sigma} \mid c_{j \beta \sigma}^{\dagger}\right\rangle\right\rangle-\sum_{b \in C u} t_{i b}^{\prime}\left(\left\langle n_{i l \bar{\sigma}}\right\rangle\left\langle\left\langle d_{b \sigma} \mid c_{j \beta \sigma}^{\dagger}\right\rangle\right\rangle+2\left\langle d_{b \bar{\sigma}}^{\dagger} c_{i l \bar{\sigma}}\right\rangle\left\langle\left\langle c_{i \alpha \sigma} \mid c_{j \beta \sigma}^{\dagger}\right\rangle\right\rangle\right) .
\end{aligned}
$$

* yunsong@bnu.edu.cn

1 Y. Kamihara, H. Hiramatsu, M. Hirano, R. Kawamura, H. Yanagi, T. Kamiya, and H. Hosono, J. Am. Chem. Soc. 128, 10012 (2006).

2 Y. Kamihara, T. Watanabe, M. Hirano, and H. Hosono, J. Am. Chem. Soc. 130, 3296 (2008).

${ }^{3}$ X. Chen, P. Dai, D. Feng, T. Xiang, and F.-C. Zhang, Nat. Sci. Rev. 1, 371 (2014).

4 C. W. Chu, Nat. Phys. 5, 787 (2009).

5 P J Hirschfeld, M M Korshunov, and I I Mazin, Rep. Prog. Phys. 74, 124508 (2011).

${ }^{6}$ H. Alloul, J. Bobroff, M. Gabay, and P. J. Hirschfeld, Rev. Mod. Phys. 81, 45 (2009).

7 A. V. Balatsky, I. Vekhter, and J.-X. Zhu, Rev. Mod. Phys. 78373 (2006).

8 N. Ni, A. Thaler, J. Q. Yan, A. Kracher, E. Colombier, S. L. Budko, P. C. Canfield, and S. T. Hannahs, Phys. Rev. B 82, 024519 (2010).

9 J. Li, Y. F. Guo, S. B. Zhang, J. Yuan, Y. Tsujimoto, X. Wang, C. I. Sathish, Y. Sun, S. Yu, W. Yi, K. Yamaura, E. Takayama-Muromachiu, Y. Shirako, M. Akaogi, and H. Kontani, Phys. Rev. B 85, 214509 (2012).

10 A. J. Williams, T. M. McQueen, V. Ksenofontov, C. Felser and R. J. Cava, J. Phys.: Condens. Matter 21, 305701 (2009).

11 J. A. McLeod, A. Buling, R. J. Green, T. D. Boyko, N. A. Skorikov, E. Z. Kurmaev, M. Neumann, L. D. Finkelstein, N. Ni, A. Thaler, S. L. Bud'ko, P. C. Canfield, and A. Moewes, J. Phys: Condens. Matter 24, 215501 (2012).

12 Y. J. Yan, P. Cheng, J. J. Ying, X. G. Luo, F. Chen, H.
Y. Zou, A. F. Wang, G. J. Ye, Z. J. Xiang, J. Q. Ma, and X. H. Chen, Phys. Rev. B 87, 075105 (2013).

13 P. Cheng, B. Shen, F. Han, and H.-H. Wen, Eur. Phys. Lett. 10437007 (2013).

14 S. Ideta, T. Yoshida, I. Nishi, A. Fujimori, Y. Kotani, K. Ono, Y. Nakashima, S. Yamaichi, T. Sasagawa, M. Nakajima, K. Kihou, Y. Tomioka, C. H. Lee, A. Iyo, H. Eisaki, T. Ito, S. Uchida, and R. Arita, Phys. Rev. Lett. 110, 107007 (2013).

15 M. Merz, P. Schweiss, P. Nagel, Th. Wolf, H. v. Löhneysen, and S. Schuppler, arXiv:1306.4222

16 A. F. Wang, J. J. Lin, P. Cheng, G. J. Ye, F. Chen, J. Q. Ma, X. F. Lu, B. Lei, X. G. Luo, and X. H. Chen, Phys. Rev. B 88, 094516 (2013).

17 S. T. Cui, S. Kong, S. L. Ju, P. Wu, A. F. Wang, X. G. Luo, X. H. Chen, G. B. Zhang, and Z. Sun, Phys. Rev. B 88, 245112 (2013).

18 T.-W. Huang, T.-K. Chen, K.-W. Yeh, C.-T. Ke, C. L. Chen, Y.-L. Huang, F.-C. Hsu, M.-K. Wu, P. M. Wu, M. Avdeev, and A. J. Studer, Phys. Rev. B 82, 104502 (2010).

19 T. Berlijn, C.-H. Lin, W. Garber, and W. Ku, Phys. Rev. Lett. 108, 207003 (2012).

20 T. Miyake, K. Nakamura, R. Arita, and M. Imada, J. Phys. Soc. Jpn. 79, 044705 (2010).

21 M. Nakajima, S. Ishida, T. Tanaka, K. Kihou, Y. Tomioka, T. Saito, C. H. Lee, H. Fukazawa, Y. Kohori, T. Kakeshita, A. Iyo, T. Ito, H. Eisaki, and S. Uchida, Sci. Rep. 45873 (2014).

22 P. Werner, M. Casula, T. Miyake, F. Aryasetiawan, A. J. Millis, and S. Biermann, Nat. Phys. 8, 331 (2012). 
23 D.-Y. Liu, Y.-M. Quan, D.-M. Chen, L.-J. Zou, and H. Q. Lin, Phys. Rev. B 84, 064435 (2011).

${ }^{24}$ L. de Medici, S. R. Hassan, M. Capone, and X. Dai, Phys. Rev. Lett. 102, 126401 (2009).

25 L. de Medici, G. Giovannetti, and M. Capone. Phys. Rev. Lett. 112, 177001 (2014).

26 Y.-M. Quan, L.-J. Zou, D.-Y. Liu, and H. Q. Lin, J. Phys. Conden. Matt. 24, 085603 (2012).

27 K. Koepernik and H. Eschrig, Phys. Rev. B 59, 1743 (1999).

28 J. P. Perdew and Y. Wang, Phys. Rev. B 45, 13244 (1992).

29 P. Soven, Phys. Rev. 156, 809 (1967).

30 D. W. Taylor, Phys. Rev. 156, 1017 (1967).

31 K. Koepernik, B. Velický, R. Hayn, and H. Eschrig, Phys. Rev. B 55, 5717 (1997).

32 M. Daghofer, A. Nicholson, and A. Moreo, Phys. Rev. B 85184515 (2012).

33 M. Daghofer, A. Nicholson, A. Moreo, and E. Dagotto, Phys. Rev. B 81, 014511 (2010).

34 T. A. Costi and A. Liebsch, Phys. Rev. Lett. 99, 236404 (2007).

35 K. Bouadim, G. G. Batrouni, and R. T. Scalettar, Phys. Rev. Lett. 102, 226402 (2009).

${ }^{36}$ H. Lee, Y.-Z. Zhang, H. O. Jeschke, R. Valentí, and H. Monien, Phys. Rev. Lett. 104, 026402 (2010).

37 D. N. Zubarev, Soviet Phys. Usp. (English Trasl.) 3, 320 (1960).

38 J. Hubbard, Proc. Roy. Soc. A 276, 238 (1963).

39 I. I. Mazin, D. J. Singh, M. D. Johannes, and M. H. Du, Phys. Rev. Lett. 101, 057003 (2008).

${ }^{40}$ K. Byczuk, W. Hofstter, and D. Vollhard, Chapter 20, 50 years of Anderson Localization ed. E. Abrahams (World Scientific, 2010).

41 D. C. Licciardello and D. J. Thouless, Phys. Rev. Lett. 35, 1475 (1975).

42 C. He, Y. Zhang, B. P. Xie, X. F. Wang, L. X. Yang, B. Zhou, F. Chen, M. Arita, K. Shimada, H. Namatame, M. Taniguchi, X. H. Chen, J. P. Hu, and D. L. Feng, Phys. Rev. Lett. 105, 117002 (2010).

43 L. Boeri, O. V. Dolgov, and A. A. Golubov, Phys. Rev. Lett. 101, 026403 (2008).

${ }^{44}$ K. Haule, J. H. Shim, and G. Kotliar, Phys. Rev. Lett. 100, 226402 (2008).

45 S. Zhou and Z. Wang, Phys. Rev. Lett. 105, 096401 (2010).

46 M. Yi, D. H. Lu, R. G. Moore, K. Kihou, C.-H. Lee, A. Iyo, H. Eisaki, T. Yoshida, A. Fujimori, and Z.-X. Shen, New J. Phys. 14073019 (2012).

47 Z.-R. Ye, Y. Zhang, B.-P. Xie, and D.-L. Feng, Chin. Phys. B 22, 087407 (2013).
48 J. Knolle, V. B. Zabolotnyy, I. Eremin, S. V. Borisenko, N. Qureshi, M. Braden, D. V. Evtushinsky, T. K. Kim, A. A. Kordyuk, S. Sykora, Ch. Hess, I. V. Morozov, S. Wurmehl, R. Moessner, and B. Buchner, Phys. Rev. B 86, 174519 (2012).

${ }^{49}$ L. X. Yang, Y. Zhang, H. W. Ou, J. F. Zhao, D. W. Shen, B. Zhou, J. Wei, F. Chen, M. Xu, C. He, Y. Chen, Z. D. Wang, X. F. Wang, T. Wu, G. Wu, X. H. Chen, M. Arita, K. Shimada, M. Taniguchi, Z. Y. Lu, T. Xiang, and D. L. Feng, Phys. Rev. Lett. 102, 107002 (2009).

50 H. Ding, P. Richard, K. Nakayama, K. Sugawara, T. Arakane, Y. Sekiba, A. Takayama, S. Souma, T. Sato, T. Takahashi, Z. Wang, X. Dai, Z. Fang, G. F. Chen, J. L. Luo, and N. L. Wang, Eur. Phys. Lett. 83, 47001 (2008).

${ }^{51}$ G. Lee, H. S. Ji, Y. Kim, C. Kim, K. Haule, G. Kotliar, B. Lee, S. Khim, K. H. Kim, K. S. Kim, K.-S. Kim, and J. H. Shim, Phys. Rev. Lett. 109, 177001 (2012).

52 J. Ferber, K. Foyevtsova, R. Valentí, and H. O. Jeschke Phys. Rev. B 85, 094505 (2012).

${ }^{53}$ L Y Xing, H Miao, X C Wang, J Ma, Q Q Liu, Z Deng, H Ding and C Q Jin, J. Phys.: Condens. Mat. 26, 435703 (2014).

54 S. F. Wu, P. Richard, A. van Roekeghem, S. M. Nie, H. Miao, N. Xu, T. Qian, B. Saparov, Z. Fang, S. Biermann, Athena S. Sefat, and H. Ding, Phys. Rev. B 91, 235109 (2015).

55 D. J. Singh, Phys. Rev. B 79, 153102 (2009).

56 V. K. Anand, P. K. Perera, A. Pandey, R. J. Goetsch, A. Kreyssig, and D. C. Johnston, Phys. Rev. B 85, 214523 (2012).

57 R. Kraus, V. Bisogni, L. Harnagea, S. Aswartham, S. Wurmehl, G. Levy, I. S. Elfimov, B. Buchner, G. A. Sawatzky, and J. Geck, Phys. Rev. B 87, 134516 (2013).

58 A. L. Efros and B. I. Shklovskii, J. Phys. C: Solid State Phys. 8. L49 (1975)

59 Y. Song, S. Bulut, R. Wortis, and W. A. Atkinson, J. Phys.: Condens. Matter 21, 385601 (2009).

${ }^{60}$ Q. Luo, G. Martins, D. X. Yao, M. Daghofer, R. Yu, A. Moreo, and E. Dagotto, Phys. Rev. B 82, 104508 (2010).

61 B. R. Kubo, J. Phys. Soc. Jpn. 12, 570 (1957).

62 P. A. Lee and D. S. Fisher, Phys. Rev. Lett. 47, 882 (1981).

63 A. Wang, Single crystal growth and physical properties of iron-based superconductors with alkali metal, Ph.D. thesis, University of Science and Technology of China, 2014.

64 W. Nolting, Chapter 4, Fundamentals of Many-body Physics: Principles and Methods, (Springer-Verlag, Berlin, 2009). 